\title{
During vertebrate development, arteries exert a morphological control over the venous pattern through physical factors
}

\author{
Alia Al-Kilani, ${ }^{1}$ Sylvie Lorthois, ${ }^{2}$ Thi-Hanh Nguyen, ${ }^{3}$ Ferdinand Le Noble, ${ }^{4}$ Annemiek Cornelissen, ${ }^{1}$ Mathieu Unbekandt, ${ }^{1}$ \\ Olena Boryskina, ${ }^{1}$ Loïc Leroy, ${ }^{1}$ and Vincent Fleury ${ }^{1, *}$ \\ ${ }^{1}$ Groupe Matière Condensée et Matériaux, Université de Rennes 1, Campus de Beaulieu, Bâtiment 13A, 35042 Rennes, France \\ ${ }^{2}$ Institut de Mécanique des Fluides de Toulouse, CNRS UMR 5502, Allées du Professeur C. Soula, 31400 Toulouse Cedex, France \\ ${ }^{3}$ Laboratoire de Physique de la Matière Condensée, Ecole Polytechnique, 91128 Palaiseau Cedex, France \\ ${ }^{4}$ Laboratory for Angiogenesis and Cardiovascular Pathology, Max Delbrück Centrum für Molekulare Medizin, \\ Robert Rössle Strasse 10, 13125 Berlin-Buch, Germany
}

(Received 3 December 2007; published 15 May 2008)

\begin{abstract}
The adult vasculature is comprised of three distinct compartments: the arteries, which carry blood away from the heart and display a divergent flow pattern; the capillaries, where oxygen and nutrient delivery from blood to tissues, as well as metabolic waste removal, occurs; and the veins, which carry blood back to the heart and are characterized by a convergent flow pattern. These compartments are organized in series as regard to flow, which proceeds from the upstream arteries to the downstream veins through the capillaries. However, the spatial organization is more complex, as veins may often be found paralleling the arteries. The factors that control the morphogenesis of this hierarchically branched vascular network are not well characterized. Here, we explain how arteries exert a morphological control on the venous pattern. Indeed, during vertebrate development, the following transition may be observed in the spatial organization of the vascular system: veins first develop in series with the arteries, the arterial and venous territories being clearly distinct in space (cis-cis configuration). But after some time, new veins grow parallel to the existing arteries, and the arterial and venous territories become overlapped, with extensive and complex intercalation and interdigitation. Using physical arguments, backed up by experimental evidence (biological data from the literature and in situ optical and mechanical measurements of the chick embryo yolk-sac and midbrain developing vasculatures), we explain how such a transition is possible and why it may be expected with generality, as organisms grow. The origin of this transition lies in the remodeling of the capillary tissue in the vicinity of the growing arteries. This remodeling lays down a prepattern for further venous growth, parallel to the existing arterial pattern. Accounting for the influence of tissue growth, we show that this prepatterned path becomes favored as the body extends. As a consequence, a second flow route with veins paralleling the arteries (cis-trans configuration) emerges when the tissue extends. Between the cis-cis and cis-trans configurations, all configurations are in principle possible, and self-organization of the vessels contributes to determining their exact pattern. However, the global aspect depends on the size at which the growth stops and on the growth rate.
\end{abstract}

DOI: 10.1103/PhysRevE.77.051912

\section{INTRODUCTION}

The main function of the blood vascular system in higher vertebrates is to transport oxygen and nutrients to cells in the peripheral tissue. The vascular system is thereby necessary for organ growth and expansion. Without a proper vascular system expansion, body size is limited due to the imbalance in oxygen delivery, which finally causes death. The vascular system is comprised of three distinct compartments: the arteries, which carry blood away from the heart through a divergent arborescence; the capillaries, where oxygen and nutrient delivery from blood to tissues, as well as metabolic waste removal, occurs; and the veins, which carry blood back to the heart through a convergent arborescence. These compartments are organized in series as regard to flow, which proceeds from the upstream arteries to the downstream veins through the capillaries. However, the spatial organization is more complex, as arterial and venous networks intercalate and interdigitate, with veins often observed

\footnotetext{
*To whom correspondence should be addressed. vincent.fleury@univ-rennes1.fr.
}

paralleling the arteries. This parallelism is in many regions absolutely obvious with veins contiguous to the arteries (Fig. 1), although in numerous instances the veins remain parallel, but at some distance from the arteries.

A largely open problematic in the field of vascular development is to understand how such a network can evolve morphologically and how design and function cross talk to create the vascular architecture. Indeed, many basic questions remain unanswered, including what cues guide vessel positioning relative to other tissues or organs, how vessel interconnection is determined, and how arterial and venous vessels form juxtaposed yet distinct and separate networks [1]. An additional unanswered question regards the relative contribution of intrinsic (molecular) programming and extrinsic (physiological or epigenetic) factors, such as hemodynamic pressure, shear stress, or interstitial stress, on the morphological evolution of the vascular system $[2,3]$.

In the present paper, we explain how arteries exert a morphological control over the venous pattern through extrinsic factors. Indeed, during vertebrate development, the following transition may be observed in the spatial organization of the vascular system: veins first develop in series with the arteries, the arterial and venous territories being clearly distinct in 


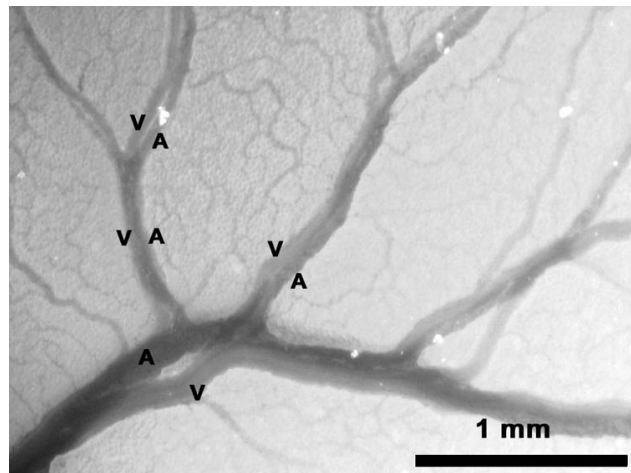

FIG. 1. Yolk-sac vessels at day 4, observed by reflection illumination: Example of veins (V) contiguous to arteries (A). Veins may appear on either side of the artery under study. Note that the main artery and vein both branch to form parallel secondary arteries and venules, which themselves branch to form even smaller parallel arteries and veins.

space. But after "some time," new veins grow parallel to the existing arteries, and the arterial and venous territories become overlapped, with extensive and complex intercalation and interdigitation. Using physical arguments, we explain how such a transition is possible and why it may be expected with generality, as organisms grow. These arguments are based on biological data from the literature as well as on in situ optical and mechanical measurements, in particular high-precision measurements of the tissue deformability. These measurements have been performed in the chick embryo yolk-sac and midbrain developing vasculatures, which have been chosen as case studies because the yolk-sac (e.g., the avian equivalent of the mammal placenta) is twodimensional and extra-embryonic and the brain is threedimensional and inside the embryo body. ${ }^{1}$

After an overview of the morphological development of the vascular system (Sec. III), the physical arguments demonstrating how arteries exert a morphological control over the venous pattern will be presented and backed up by relevant biological data, some of which have already been described in Le Noble et al. [4] (Sec. IV). These arguments will also be supported by simple theoretical or computational models describing the flow pattern in a vascular network (Sec. IV). The results will finally be discussed in light of the current knowledge of the molecular distinction between arteries and veins (Sec. V). The experimental methodologies we refer to throughout the paper, as well as the computational modeling approach used in Sec. IV, will be presented first (Sec. II).

\section{MATERIALS AND METHODS}

Embryonic material. Fertilized chick (Gallus gallus, brown leghorn) embryos were purchased from Couvoir de

\footnotetext{
${ }^{1}$ It is sometimes emphasized that the yolk-sac circulation is singular because its function is to absorb nutritive material at capillary level, this material being subsequently conveyed to the embryo via the yolk-sac veins. Thus, it works in the opposite way than the embryo internal vasculature, where nutriments are transported by the arterial blood and delivered to the organs at capillary level.
}

l'Ausier in Louvigné du désert (Ille et Vilaine, France) and incubated at $38{ }^{\circ} \mathrm{C}$ in a humidified atmosphere (standard egg incubator). The chicken embryos were handled and disposed following the French regulations for avian in-shell embryos. All embryos were disposed within one week of development.

In vivo Imaging. For in vivo imaging, embryo New cultures [5], or eggs containing a window [6], were placed between two glass heating plates (Minitüb HT300, Germany), the lower one at $37{ }^{\circ} \mathrm{C}$, the upper one at $39{ }^{\circ} \mathrm{C}$ to avoid condensation. Embryos were observed by reflection optical microscopy using a Leica MZFLIII stereomicroscope equipped with a digital camera $(1024 \times 800$, 8-bit color Scion corporation camera). In a few cases, indicated in figure legends, the yolk-sac was rinsed and transferred to a glass slide for direct observation by transmission illumination using the same microscope. Images were acquired using $\mathrm{NIH}$ Image in its ImageJ version. The vasculature was visualized with a $1-14 \times$ zoom. Assignment of artery or vein identity to a particular vessel was made based on the direct observation of the flow direction of blood cells transiting the vessel. Alternatively, surface topography of the embryos was observed by shadowgraph, in which case an OPTEM microscope was used. A sketch of this experimental technique and further explanations are given in Fig. 2 and the legend.

Air-puff tonometry. In order to measure the deformability of the tissue surrounding arteries, we have designed an airpuff tonometer (APT). The principle of the instrument is the following: a small air puff is blown through a micropipette and impacts the surface at a $45^{\circ}$ angle. The size of the area deformed by the air puff, which increases when local deformability increases, is measured optically by following the displacement and/or size variation of a light spot shone on the deformed area. The spot position and size vary because the spot is initially oriented slightly offset from the direction of specular reflection. As the surface flexes down, the spot center moves while the spot is deformed. For more details on the technique as well as on its calibration, see Fig. 3 and the legend. The resolution of the APT is in principle equal to the injection diameter of the air puff. In a first series of experiments, samples (rinsed yolk-sacs lying flat on a Petri dish) were placed on a moving table (Newport) so as to scan the surface with the APT at a speed of $0.1 \mathrm{~mm} / \mathrm{s}$ and with a data acquisition rate of $5 \mathrm{~Hz}$. In this first series of experiments, the injection diameter was $20 \mu \mathrm{m}$. Using this "scanningmode" procedure, deformability gradients are directly recorded, although detailed information about the localized mechanical behavior cannot be obtained. In a second series of experiments, the samples (rinsed yolk-sacs lying flat on a Petri dish) were mounted on a goniometer to provide for tilt angle correction. The deformability was measured in situ by turning the air on and recording the time evolution of the deformation trough. Using this "local-mode" procedure, detailed information on the surface reaction to a localized force is obtained. In this second series of experiments, the injection diameter was $50 \mu \mathrm{m}$.

Image processing and analysis. The images obtained by reflection optical microscopy, a combination of yellow from the yolk-sac and red from the red blood cells, were posttreated using ImageJ. To maximize the contrast produced by the red blood cells, which mark the blood vessels localiza- 


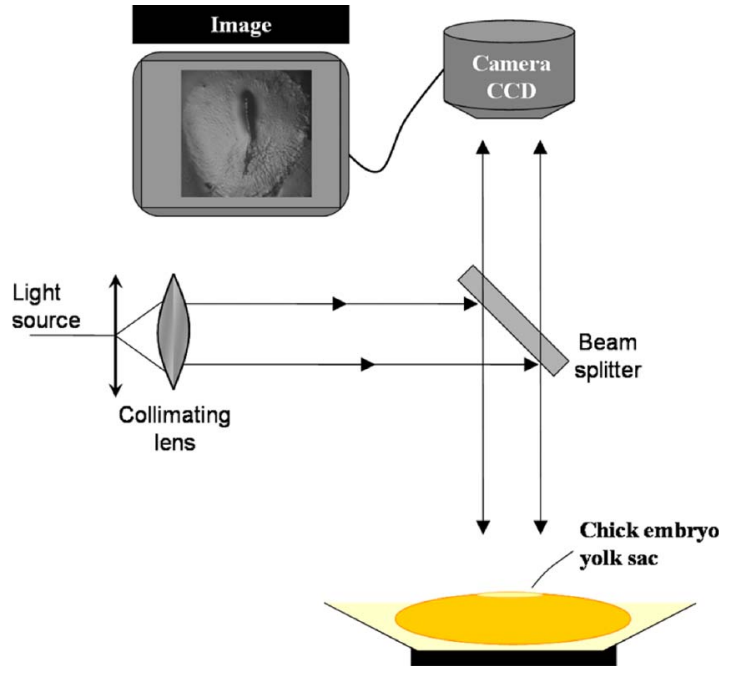

FIG. 2. (Color online) Scheme of the shadowgraph setup used to map the surface topography of the growing yolk-sac. A parallel beam of light is shone on the surface of the yolk-sac, and the reflection is observed in the direction of specular reflection. Since the embryo is not flat, there is only one direction of observation, which may be difficult to find. Once this direction of specular reflection has been found, details of the surface appear as brighter or darker, depending on the surface curvature. A small concave feature, if approximated by a paraboloid, displays a bright spot in the center of a dark halo. Inversion of colors, as done in Fig. 9, right, allows recovering the natural impression of colors (bumps appear bright whereas fissures appear dark). By this mean, the details of the surface topography are evidenced. When the subject is not observed under a strict specular reflection, interesting shadowing effects may be obtained, which reveal the surface corrugations. Note that this technique is often used by physicists to explore refraction index gradients. For example, shining a parallel light through a warmed gas reveals temperature gradients and flows.

tion, and to filter the yolk-sac yellow background, the red and blue components of these RGB (Red Green Blue) colored images were discarded. Their green component was converted to black and white images, whose contrast was adjusted. In order to estimate the number of red blood cells traveling in a given area surrounding a particular vessel, the gray-level profile, averaged along the direction parallel to the vessel in this area, was plotted as a function of the distance to the vessel.

Numerical simulations of the wall shear-stress spatial distribution in a vascular network. An extremely simplified model of vascular network is that of a bidimensional lattice of tubes of given radii $r_{i}$ and lengths $l_{i}$, represented by a specified connectivity matrix. The flow $Q_{i}$ through each tube is related to the pressure drop $\Delta P_{i}$ by Poiseuille's law $\Delta P_{i}$ $=R_{i} Q_{i}$ where $R_{i}$ is the tube hydrodynamic resistance given by $R_{i}=8 \mu l_{i} / \pi r_{i}^{4}$. In this expression, $\mu$ is the viscosity of blood, considered as a Newtonian fluid (viscosity $1.2 \mathrm{cP}$ ). The application of Poiseuille's law to each tube in the network, along with the conservation of mass at each node, leads to a set of linear equations. Given the pressure difference across the network arterial entry and venous exit points (50 $\mathrm{mm} \mathrm{Hg}$ ), these equations can be solved to yield the pressure at each node and, hence, the flow as well as the wall shear stress in each tube. In this way, the shear-stress variations due to the remodeling of specific vessels, whose radii are modified from $r_{i}$ to $r_{i}^{\prime}$, can be studied.

\section{MORPHOLOGICAL DEVELOPMENT OF THE VASCULAR SYSTEM}

In general terms, vascular networks form by the remodeling of an existing meshwork of small capillaries, called a capillary plexus. This means that an initial meshwork of small capillary vessels progressively evolves into a complex hierarchical branching structure, in which all the large vessels, be it arteries or veins, were once vessels of the smallest size [7]. In general terms also, there are three ways of formation of capillary vessels: either in situ (de novo) (for example, during the early phases of embryonic development) or from existing capillaries which emit sprouts ("sprouting") or which are split in two along their axis ("intussusception"). More specifically, in the de novo formation of capillary vessels, dispersed blood islands (i.e., aggregates of hematopoietic cells, the precursors of red blood cells, surrounded by endothelial cells, the constitutive cells of the capillary wall) first form. The endothelial cells surrounding these blood islands soon coalesce and anastomose to form a capillary meshwork, called the primary capillary plexus, which serves as a scaffold for the beginnings of circulation [8,9]; see Fig. 4 , left and middle. De novo formation is typically observed in growing embryos, where the first capillary meshworks have been laid down before the onset of heart beat, around $24 \mathrm{~h}$ in the chick. After the onset of heart beat and blood perfusion, the yolk-sac capillary plexus is rapidly remodeled into arteries and veins and a functional circulatory loop is established [4,10]; see Fig. 4, right. Such a remodeling is largely linked to hemodynamic forces, in particular the wall shear stress, such that vessels which undergo a high flow are more likely to enlarge [4,11-13], in a self-organized fashion [14]. When the blood circulation fails to establish (for example, after heart removal), the yolk-sac capillary plexus fails to remodel, although continuing to grow $[4,15]$. Note that several larger vessels (e.g., the dorsal aorta and the posterior cardinal vein) have already developed before the onset of perfusion. However, these vessels do not constitute a circulatory loop, and flow is still needed to establish patent connections between them [1]. Thus, even if the details of mechanotransduction and of its nonlinearities as well as the exact mechanism of vessels remodeling are still debated $[2,3,16,17]$, it is now well accepted that the formation of a functional circulatory loop requires at least two fundamental features: first, a capillary plexus and, second, a flow.

However, when described carefully, the physiological formation of a vascular system exhibits a striking spatiotemporal feature, which is rarely emphasized in the literature. At the very beginning of the remodeling process, the remodeling of the capillary plexus starts from a point close to the entry of the flow (e.g., in the case of the yolk-sac, a point, the presumptive navel, where the flow exits the embryo and enters the yolk-sac) and extends centrifugally (i.e., along the proximal to distal direction). As a consequence, arteries are the first large vessels to appear and radiate away from the 

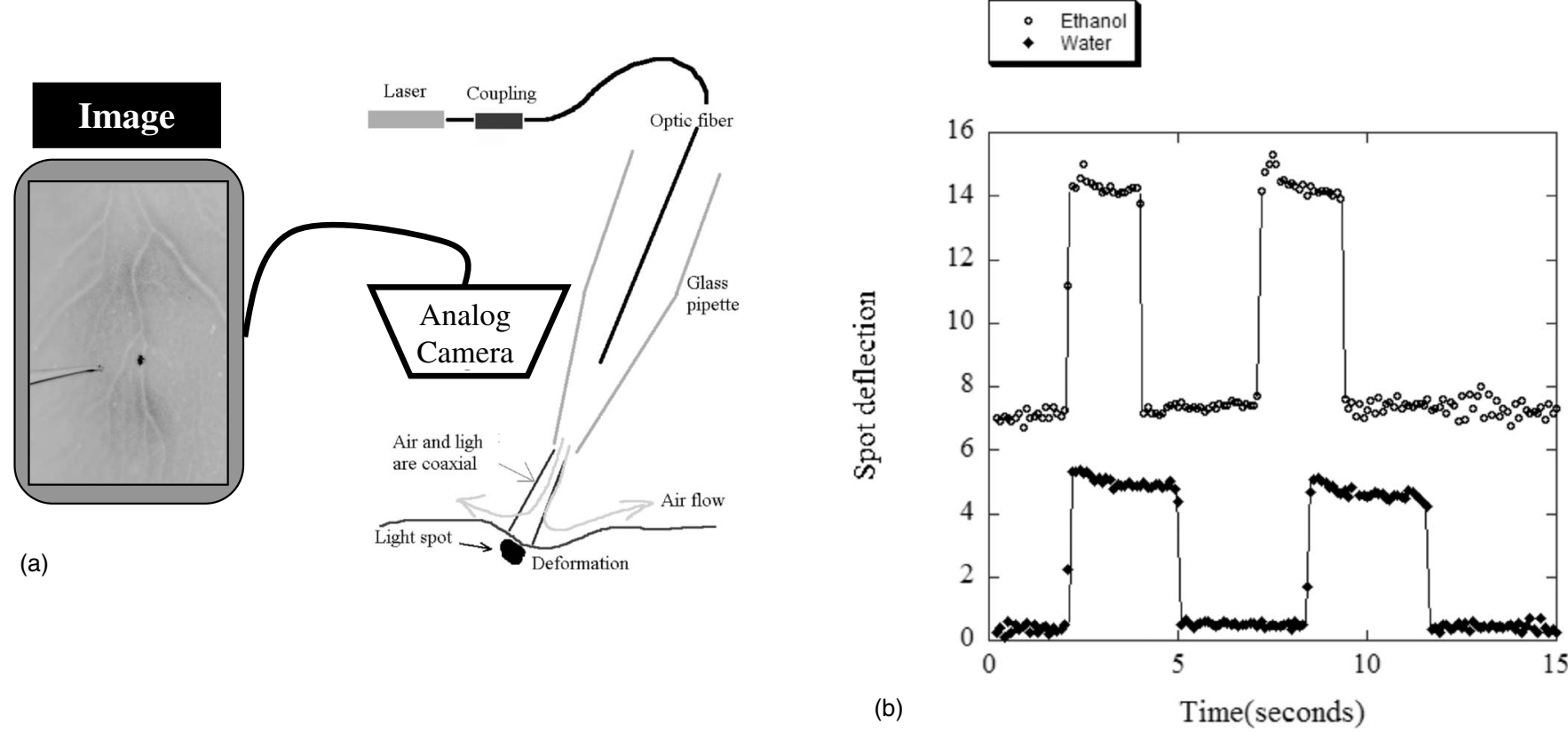

FIG. 3. Air-puff tonometry: principle [Fig. 3(a)] and calibration using water and ethanol [Fig. 3(b)]. (a): Scheme of the air-puff tonometer used to map the local deformability of the growing yolk-sac. An air puff (approximately $10 \mathrm{cc} / \mathrm{min}$ ) is blown through a $20-$ or $50-\mu \mathrm{m}$ micropipette made using a pipette puller (Sega) and impacts the yolk-sac surface at a $45^{\circ}$ angle. The pipette is mounted on a motorized rotating stage (Newport) to provide for tilt angle correction. The size of the area deformed by the air puff, which increases when the local deformability increases, is measured optically. For that purpose, the pipette has been modified to become the lighting element of the optical setup. Its end was first metallized by copper physical vapor deposition and additionally painted in order to guide the light and to ensure glass opacity. Then, the cleaved and bared end of a multimode optical fiber "working fiber" (HCP MO200T from SEDI) was introduced inside the pipette and inserted as deep as possible without obstructing the air flow path. The other end of the optical fiber was coupled to an "injection fiber" using a splice device (Siemon ULTRAsplice U.S.-250). At last, a laser beam produced by a diode (12 mW Melles Griot) was injected in the "injection fiber" using an optic positioner FP-1A and a bare fiber chuck holder FPH-J (Newport). The light spot thus emitted by the micropipette falls inside the deformation trough. Depending on the absolute angle of the pipette tip with respect to the surface, either the spot size or the spot position changes when the turning the air on, generally both. This can be adjusted by having the pipette shoot more or less ahead of the air jet. The spot is observed at right angle with an analog video camera (Watek 512) which continuously films the surface and records on line its size and displacement (with a frame grabber from SCION corporation interfaced with NIH-Image). The light spot appears in dark due color coding inversion. (b) APT calibration with respect to reference surfaces (water and ethanol). The figure shows the comparison between the spot deflections (in pixel units, corresponding to approximately $4 \mu \mathrm{m} /$ pixels) obtained on ethanol and water surfaces, in otherwise exactly the same conditions, as a function of time. At the moment when the air is turned on, the spot is strongly deflected and it returns to its original position when the air is turned off. The data are offset for clarity. As expected, the ethanol surface is significantly more deformable than the water surface, the spot deflection for ethanol being about twice the deflection for water. Indeed, in this regime, where gravity effects are negligible compared to surface tension effects (i.e., negligible Bond number), the maximal penetration depth of the air jet into the liquid surface should be inversely proportional to the surface tension of the liquid [39]. Here, the water/ethanol surface tension ratio is of order 3 .

flow entry point toward the distal end of the growing organ. The veins appear later, when the capillary plexus remodels along the blood return path, from a point close to the exit of the flow. Subsequently, they also radiate along the proximal to distal direction. Thus, the blood flow loops from the arteries to the veins through the distal capillary plexus and the veins are initially in series with the arteries. At this stage, the arterial and venous territories are clearly distinct and nonoverlapping in space, as evidenced by the distinct localization of their specific molecular markers ${ }^{2}$ [18]. This configuration is called the cis-cis configuration, or primary

\footnotetext{
${ }^{2}$ Arterial and venous endothelial cells are molecularly distinct. For example, arterial cells express a transmembrane protein, called ephrin-B2, which is absent in the venous cells.
}

circulation [15]. The cis-cis loop is very conspicuous in the early yolk-sac of the chicken embryo, for example, where it includes, in series, the usual vitelline arteries and cranial and caudal veins patterns [Fig. 5(a)]. The cis-cis loop may also be observed, although in three dimensions, in the brain [Fig. $5(\mathrm{~b})]$.

But even if the circulatory loop seems to be well established, the venous pattern starts suddenly to change, such that after this change, a completely new set of veins has appeared, the pattern of which is parallel to the arterial pattern. The parallelism is sometimes so perfect that highmagnification microscopy is needed to realize that what appears at first glance to be a large vessel is actually a pair of distinct tubes, with flows in opposite directions [Figs. 1 and $6(\mathrm{a})]$. In the case of the chicken yolk-sac, this new configuration starts to develop around 3.5 days and proceeds from 


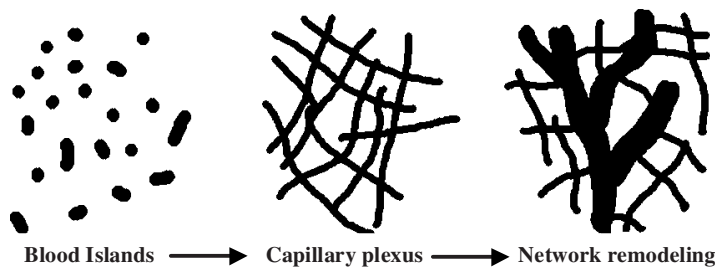

FIG. 4. Schematic description of the different steps leading to the formation of a vascular system: blood islands (aggregates of hematopoietic cells, the precursors of red blood cells, surrounded by endothelial cells, the constitutive cells of the capillary wall) form during the early phases of embryonic development. Following their formation, endothelial cells surrounding these blood islands soon anastomose to form a capillary meshwork, called the primary capillary plexus, which serves as a scaffold for the beginnings of circulation. The capillary plexus is subsequently remodeled into a complex hierarchical branching structure, including arteries and veins. Note that in this remodeling process, all large vessels, be it arteries or veins, were once capillaries of the smallest size. For more details, see [10].

proximal [Fig. 6(a)] to distal areas [Fig. 6(b)]. One very obvious testimony of this transition is that, after four days, the umbilical chords on each side of the embryo consist of joined arteries and veins, while in the previous flow situation, the navel region only displayed the vitelline arteries [15].

The configuration in which arteries and veins are parallel, but with opposite flow directions, is called the cis-trans configuration, or secondary circulation [15]. In this configuration, the arterial and venous territories, as evidenced by the location of their molecular markers, are overlapping, with extensive and complex intercalation and interdigitation [18]. Note that in many parts of the adult body, both cis-cis and cis-trans configurations subsist, the main veins being sometimes paired to the main arteries (an explicit example are the deep "venae comitantes" of the limbs that so closely accompany an artery that its pulsatility aids venous return), but sometimes not (e.g., the superficial veins of the limbs).

In summary, as the parts of the animal grow (yolk-sac, brain, limbs, etc.), one may observe a transition, or even metamorphosis, of the vessel structure and of the flow configuration from a loop to a hairpin, as schematized in Fig. 7. How is this transition possible and to what extent it may be expected with generality are the issues we address in this paper. We show that simple physical facts, backed up by relevant biological data, make this transition unavoidable as the piece of tissue being vascularized grows.

\section{INGREDIENTS OF THE TRANSITION FROM THE PRIMARY TO THE SECONDARY CIRCULATION}

As already mentioned, it is now well accepted that the blood flow provokes a progressive enlargement of the vessels. Thus, it is logical that capillary vessels will enlarge along a loop joining the entry point of the flow to the exit point of the flow, such that eventually a loop of large vessels should be observed across the distal capillary plexus. In general terms, a path of vessels, arteries toward veins, should be found in series. However, why should this loop be unstable, such that a second set of veins grows, and why should this second set be so parallel to the arteries? In the following, the three ingredients explaining such a transition will be successively introduced.

\section{A. Growing arteries progressively disconnect from the capillary plexus}

As experimentally demonstrated by Le Noble $e t$ al. in the chick yolk-sac [4], the growing arteries progressively disconnect from the capillary plexus. Indeed, during the flowdriven remodeling process of the capillary plexus, most of the capillaries initially linked to the growing arteries are progressively cut off, as the arteries enlarge. This arterial disconnection has been initially pointed out by Fleury and Schwartz [17] who have also shown that it is necessary to allow the formation of two distinct and yet overlapping arterial and venous trees, while avoiding the direct shunting between them. It is therefore an extremely important feature of the formation of the large vessels. Note that a simple mechanistic approach describing the deformation of the mesoderm in the branching area between two vessels of dissimilar diameters is sufficient to explain such a phenomenon ${ }^{3}$ [19].

Thus, as an artery advances and enlarges across the capillary plexus, the progressive cutoff of the previously connected capillaries turns this artery into a single tube with no or only rare sidebranches [Figs. 6(b) and 8(a)-8(c)] and separates it physically from the neighboring plexus. As a consequence, its outflow moves to a more distal region. Finally, in the capillary plexus neighboring the disconnected artery, the flow direction reverses and the arterial molecular markers are down-regulated [4]. A similar scenario may be observed in the brain and the eye, and in many other parts of the body [20].

\section{B. Capillary plexus in the vicinity of the disconnected arteries is remodeled, leaving down a prepattern for further venous growth}

The cutoff of the small capillaries in the vicinity of the larger arteries creates dangling sprouts ("dead ends"), which at one side are still connected to the plexus, and, at the other side, where the flow must be zero, face the disconnected arteries [see Fig. 8(c)]. Such dangling sprouts can be observed on mercox casts of the vasculature (see Fig. 6 in [4]). Also, they can be indirectly observed using in vivo micros-

\footnotetext{
${ }^{3}$ Indeed, arterial disconnection can be studied by calculating the flow induced viscoelastic deformation of the mesoderm, considered as an incompressible elastic solid (see Sec. IV B), in a region where a side (capillary) branch is connected to a larger main (arterial or venous) branch, by a two-dimensional fluid-structure interaction solver. The closure rate of the smaller side branch both depends on the bifurcation branching angle and on the ratio of the velocities in both branches. In the case of diverging bifurcations (arterial domain), this closure rate is positive in a very large range of parameters. Thus, side branches become almost systematically disconnected from the main branch. For more details, see Fig. 11 from [19].
} 


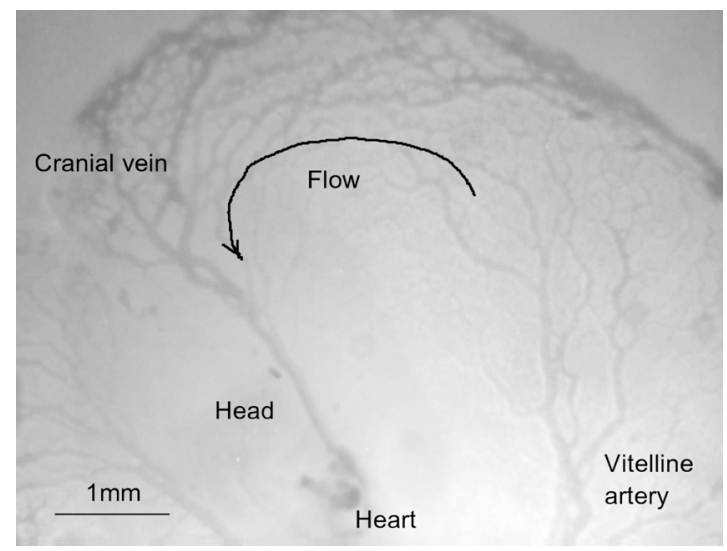

(a)
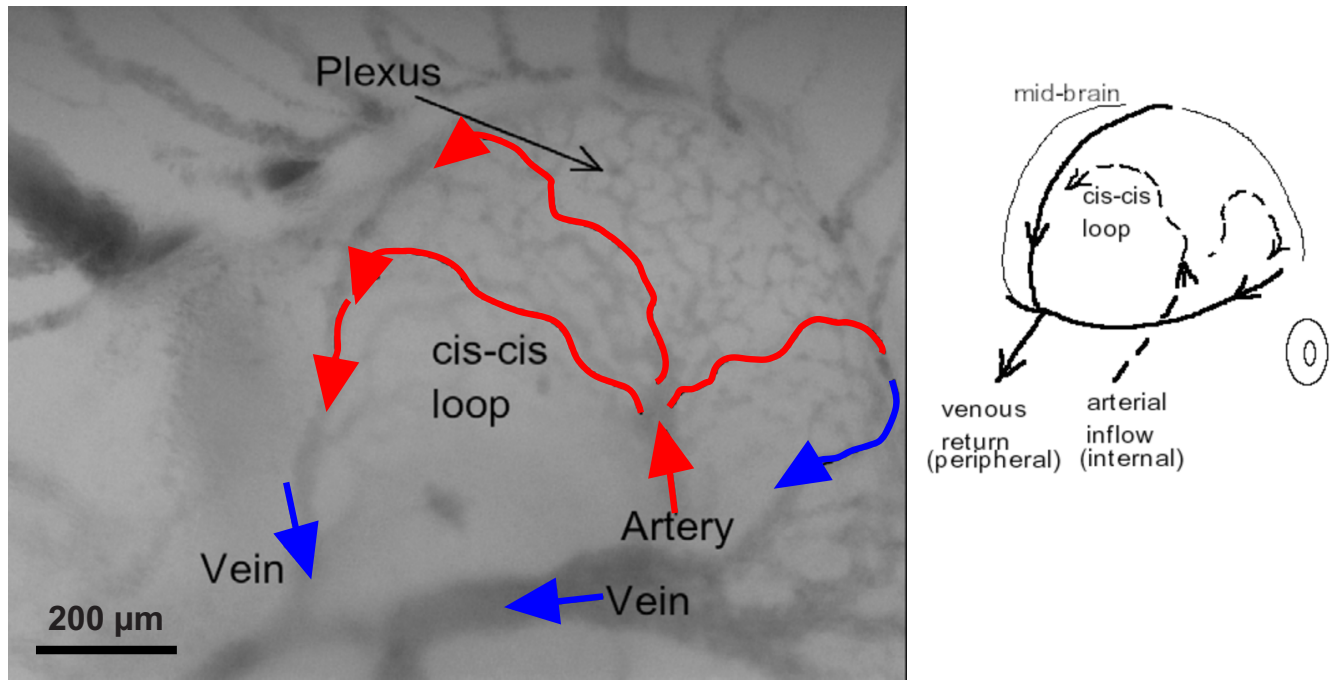

(b)

FIG. 5. (Color online) Yolk-sac (a) and brain (b) vessels in the cis-cis configuration (primary circulation stage). (a) A typical cis-cis vascular pattern in the yolk-sac at an early developmental stage (2.5-day embryo). For clarity, the heart and head are indicated. The vitelline artery is at the right, the cranial vein in the middle and elongating toward the top-left. The flow loops from the vitelline artery up to the cranial vein and back. The process of arterial disconnection (see text, Secs. IV A and IV B) starts already to be faintly visible, in the whiter and quite thin area that starts to surround the vitelline artery. (b) In vivo observation of a 3-day embryo midbrain showing the capillary plexus and the early cis-cis flow route. Note that the arterial inflow is situated deeper inside the brain shell, while veins are more superficial and appear with a better contrast. The structure of the initial flow pattern is three dimensional, whereas the yolk-sac is two dimensional. However, the topology is similar: a central arterial input flowing toward a distal venous collecting vessel located on top of the head and along the boundary between the mid-brain and forebrain.

copy in the yolk-sac because they contain pools of stagnant blood [indicated by arrows in Fig. 6(b)].

In order to gain some understanding of the mechanical properties of the growing arteries and their neighborhood during this arterial disconnection, shadowgraph and air-puff tonometry have been used. Figure 9 displays the direct in vivo observation of the yolk-sac surface topography by shadowgraph: during the disconnection process, the growing artery as well as its immediate neighborhood is swollen, suggesting a higher pressure in this swollen area. Note that, as this area includes a fluid domain (the blood contained in the vascular lumens) and an incompressible solid, although very soft, elastic domain (the living cells of the mesoderm, including the endothelial cells of the vessel walls), the pressure $p$ must be defined by $p=-\frac{1}{3} \operatorname{tr}(\sigma)$, where $\sigma$ denotes the stress tensor. This definition holds in both fluid and solid phases and will be constantly used in the following. In the fluid, $p$ corresponds to the usual hydrostatic pressure. In order to confirm the pressure increase in the swollen area detected by shadowgraph, APT has been performed. Indeed, in a porous and elastic medium (a so-called poroelastic medium), the deformation due to a prescribed external load, which can be imposed by an air puff such as in APT, must increase when the pressure in the liquid phase decreases [21].

Figure 10(a) displays a typical scanning-mode APT measurement performed at right angle from a growing artery, demonstrating the coexistence of two distinct areas: a stiff area, including the artery and its immediate neighborhood, ending about $150 \mu \mathrm{m}$ away from the artery center, and a soft area, outside. Figure 10(b) shows 12 pairs of local-mode 


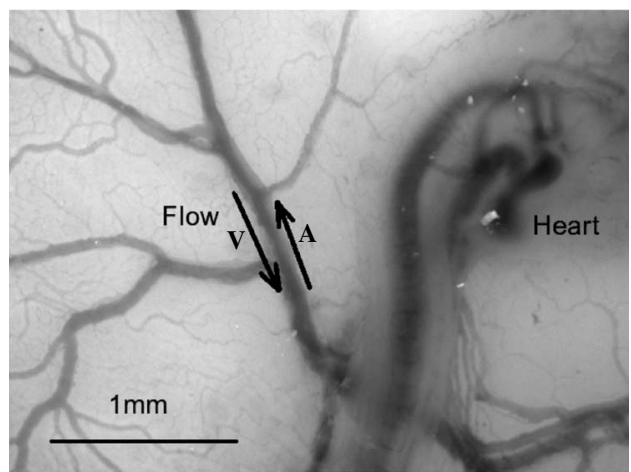

(a)

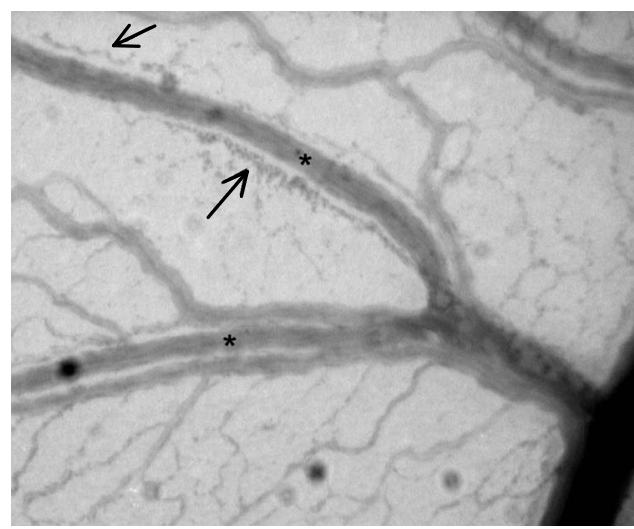

(b)

FIG. 6. Yolk-sac vessels in the cis-trans configuration (secondary circulation stage). (a) A typical cis-trans vascular pattern at a later developmental stage (4-day embryo). One must pay attention to the fact that, in (a) and (b), almost all arteries are paired with a vein. (b) A magnified view of a 4-day embryo showing the process of formation of the vein paired to each artery, in a more distal area than (a). To the sides of the arteries (stars), dangling sprouts due to capillary disconnection (see text, Sec. IV B) become discernible because they contain pools of stagnant blood (arrows).
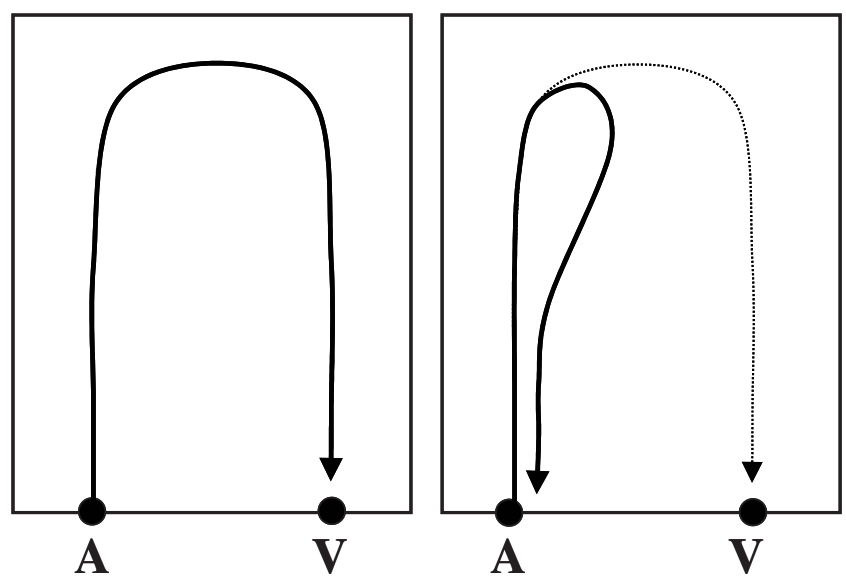

FIG. 7. Schematic description of the flow pattern in the cis-cis (left) and in the cis-trans (right) configuration. The initial arterial entry and venous exit points are indicated by $\mathrm{A}$ and $\mathrm{V}$, respectively. The formation of a venous path adjacent to the artery will lead to a flow decrease in the initial set of veins, indicated by a dotted line in the right panel, which may lead, or not, to their regression.

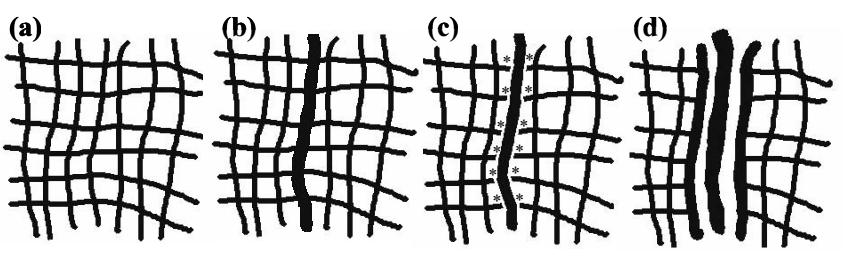

FIG. 8. Pictorial description of the formation of veins next to the arteries. First, the capillary plexus is homogeneous (a). It is then remodeled to form an arterial pathway (b). The growing artery disconnects from the plexus, leaving dangling capillaries (c), with flow stagnation areas (indicated by stars). See also Fig. 6(b), where the dangling capillaries become discernible because they contain pools of stagnant blood. The useless endothelial material is progressively excluded from the neighborhood of the artery and contributes to enlarge the capillary lumens at a short distance from the artery (d).

APT measurements performed along the growing arteries and outside the stiff area (e.g., midway between two neighboring arteries when possible). Similar data have been obtained in all the arteries which have been studied by the same technique in 20 different yolk-sacs. In a few instances, the deformability of the soft area was measurable, whereas the arterial bed was too stiff to evidence any deformation (data not shown). Thus, the results confirm a large difference of deformability between the stiff and soft areas, approaching in average a factor of 2 . As a consequence, scanning-mode as well as local-mode APT confirms the existence of a higherpressure area surrounding the growing arteries (i.e., arteries and their immediately neighboring tissue) compared to the pressure level in the tissue situated afar.

Therefore, a localized negative pressure gradient is evidenced in the tissue in the neighborhood of the growing arteries, while moving away from them, as schematized in Fig. 11. However, the yolk-sac tissue is itself a porous medium since it includes capillary vessels, which have previously been disconnected from the growing artery. As such, this deformable and incompressible porous medium is submitted to a localized negative pressure gradient oriented from the arterial bed and away from it. Thus, the fluid contained in the (capillary) pore space flows from the high-pressure region to the low-pressure region. This induces a decrease in the pore-
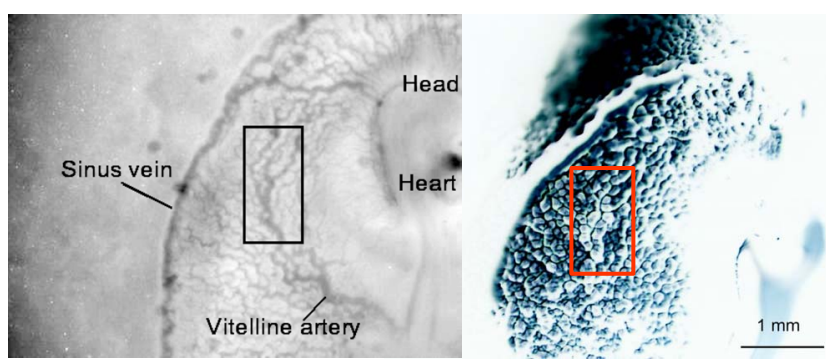

FIG. 9. (Color online) Direct in vivo imaging of the yolk-sac of a 2-day embryo still exhibiting the cis-cis configuration: surface observed by optical microscopy (left) and topography observed by shadowgraph (right). Boxes: the same young arteriole, imaged by both techniques, is highlighted. The shadowgraph reveals a swollen region around the growing arteries (right, bright areas in the red box). 

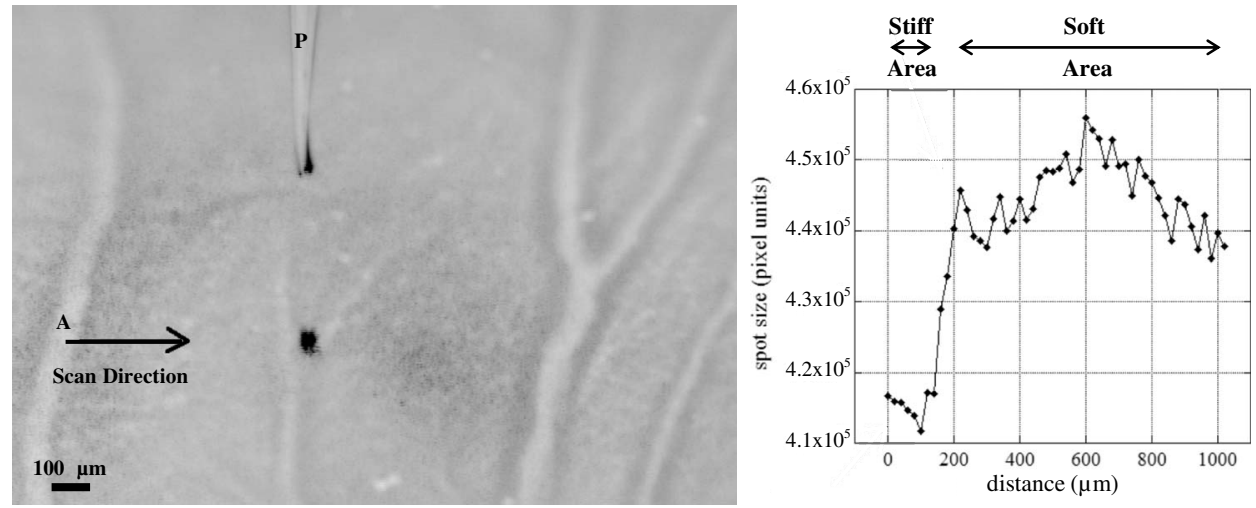

(a)
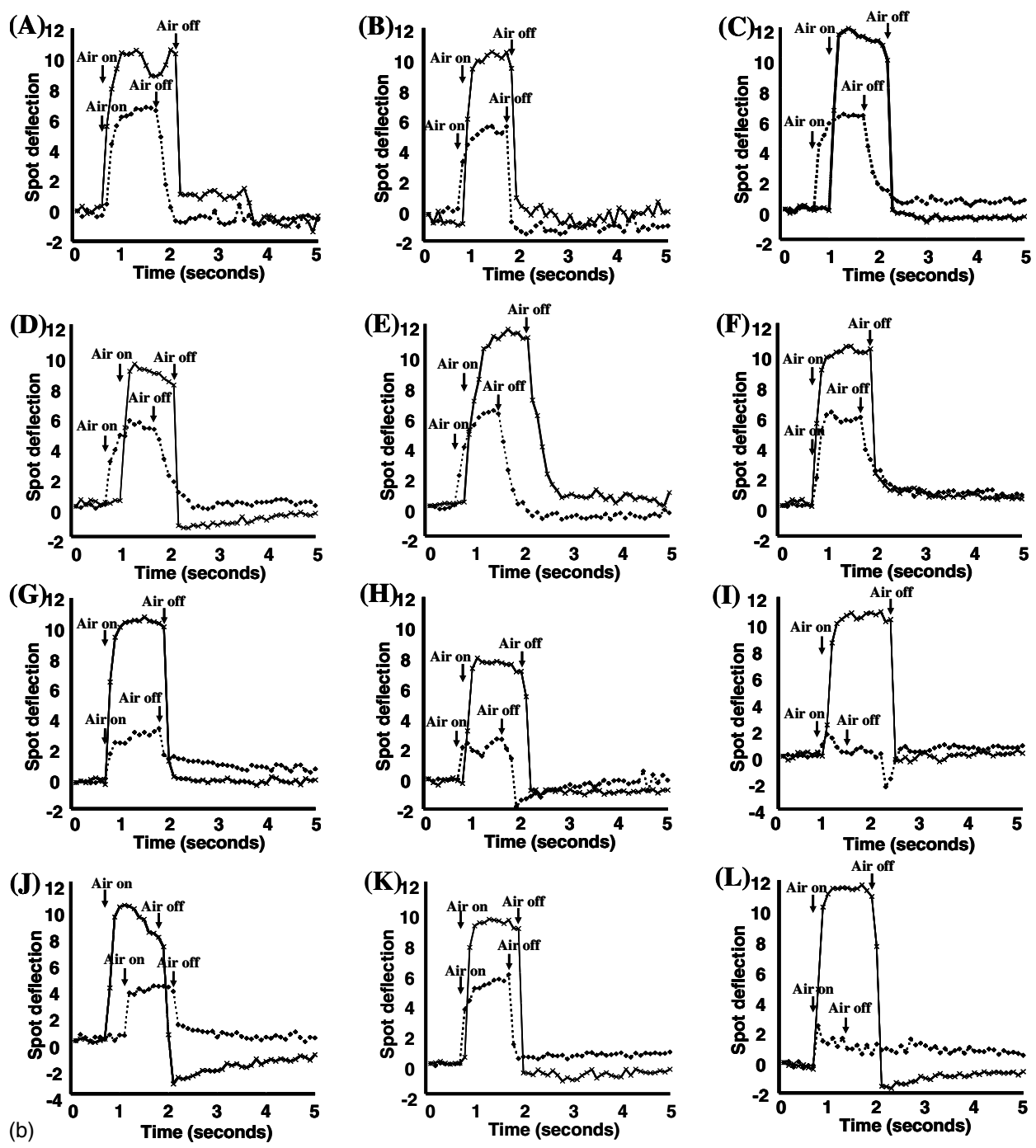

FIG. 10. Yolk-sac deformability measurements by APT in scanning mode (a) and local mode (b). (a) A typical scan performed by APT in scanning mode at right angle from a growing artery in a 4-day yolk-sac. Spot size as a function of the distance from the center of the growing artery (denoted by $\mathrm{A}$ in the left image, where $\mathrm{P}$ indicates the tip of the micropipette). In this experiment, the spot is positioned such that its size increases when the local deformability increases. Thus, APT evidences two distinct areas: a stiff area including the growing artery and its immediate neighborhood, about $150 \mu \mathrm{m}$ large from the artery center, and a softer area, outside. In this example, the vein forming in between arteries is found to follow the area of largest deformability. (b) Twelve examples of pairs of local-mode APT measurements performed along the growing arteries (dotted lines) and outside the stiff area (plain lines) of a 2.5 days yolk-sac [(A) and [(B)] and of a 4-day yolk-sac $[(\mathrm{C})-(\mathrm{L})]$. In these data, the APT spot was positioned for detection of the spot displacement (which is more accurate than the spot size). Spot deflection in pixel units $(1$ pixel $=4 \mu \mathrm{m})$ is plotted as a function of time. The time interval between each data point is $0.1 \mathrm{~s}$. 


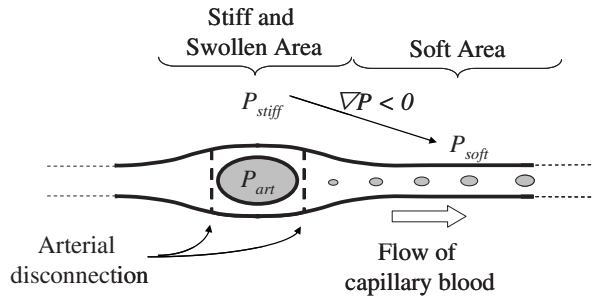

FIG. 11. Schematic representation of the yolk-sac cross section in the neighborhood of a growing artery. The fluid phase (blood) is represented in gray. The capillary lumens form an interconnected network of capillary pores whereas the arterial lumen has been previously disconnected from the capillary pore space, as indicated by the dashed lines.

space volume in the region of higher pressure: in this region, pores are squeezed like in a sponge; see Fig. 11. As a consequence, the liquid in the capillary lumens, including the red blood cells, must be progressively expelled toward the low-pressure area. This explains the displacement of the little pools of stagnant blood, described above, somewhat afar from the artery, but parallel to it. In the same fashion, based on the demonstration by Le Noble et al. [4] that no significant amount of endothelial cell apoptosis (programmed cell death) can be detected around these pools of stagnant blood, we hypothesize that the endothelial cells of the dangling sprouts previously containing this stagnant blood must also be expelled. Indeed, the existence of a specific region surrounding the arteries and devoid of capillaries as well as dangling sprouts can be experimentally evidenced by direct in vivo observations by transmission illumination on rinsed yolk-sacs (Fig. 12), which has been confirmed by labelings of the endothelial cells (data not shown) and which is supporting the above hypothesis. In the same way, an avascular region around the largest arteries can be detected by image analysis of data obtained by reflection microscopy in the brain [see Figs. 13(a) and 13(b) and legends] or in the eye (data not shown).

Finally, as no significant amount of endothelial cell apoptosis can be detected in the vicinity of the disconnected arteries, the expelled endothelial cells must have contributed to the remodeling of other vessels. If these endothelial cells are incorporated into the neighboring capillaries (i.e., the capillaries adjacent to the disconnected artery) an increase of their radius [as schematized in Fig. 8(d)], and therefore a favored path for the blood to flow, must be observed. Indeed, such a favored path, located somewhat afar from, but parallel to, an existing artery, can be directly observed in the yolk-sac [Fig. 6(b)]. It can also be detected by image analysis in quite different organs, like the brain, even in cases where no recognizable vessel is already present [see Figs. 13(a) and 13(b) and legends].

An estimate of the radius increase of the aforementioned capillaries can be made by assuming a periodic distribution of the capillaries in the plexus [Fig. 8(a)] in order to simplify the situation observed in mercox casts or by optical microscopy. Under this assumption, the quantity of extra-tissue of vascular wall corresponds to one capillary vessel in average, dangling with a spatial period of one capillary vessel [Fig. $8(\mathrm{c})]$. As the total surface of the capillary wall must approxi-
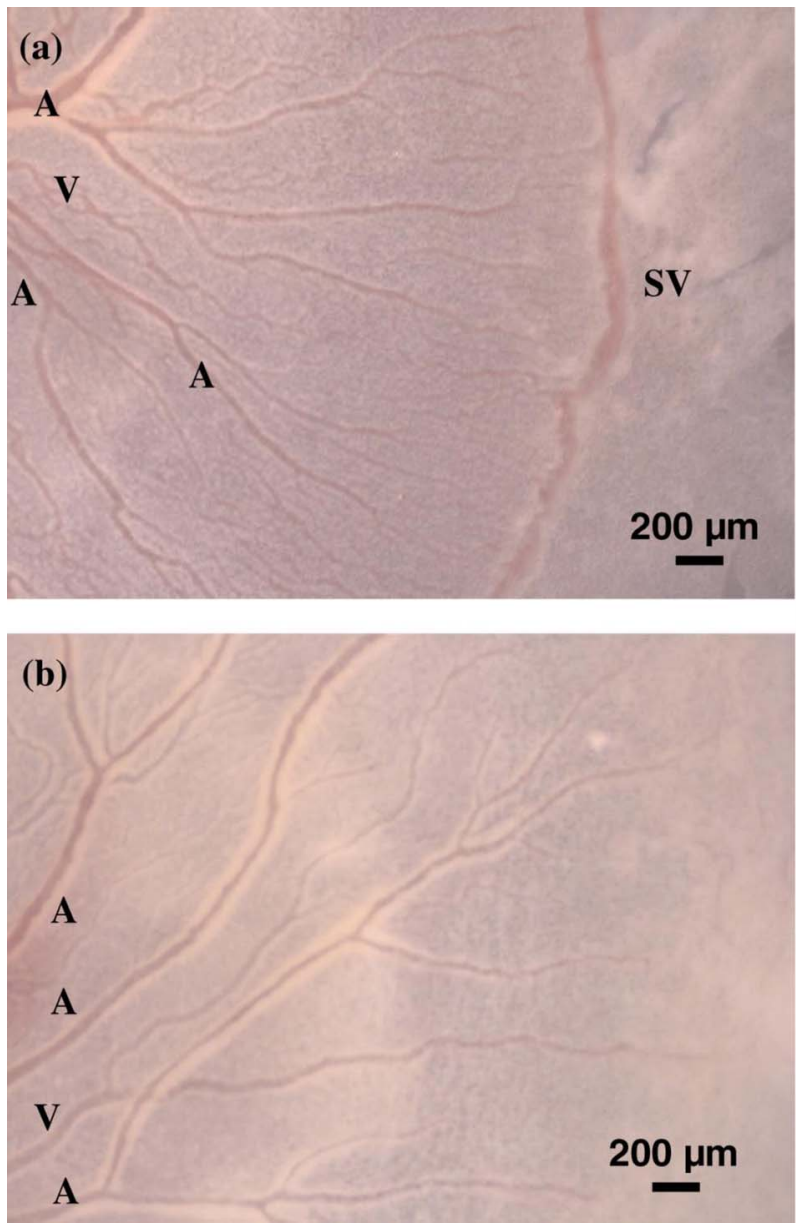

FIG. 12. (Color online) Direct in vivo observation by transmission illumination of 2-day (a) and 4-day (b) rinsed yolk-sacs: Evidence of a specific region surrounding the arteries. (a) When a yolk-sac is rinsed and transferred to a glass slide, direct observation by transmission illumination shows that there exists a region around the largest arteries, devoid of capillaries and of erythrocytes, which appears brighter in the figure. This brighter area is not observable around the distal arterioles [e.g., arterioles extending toward the sinus vein (SV) in (a)]. Indeed, arteries develop from the yolk-sac center to its periphery and, thus, the vessels which lie in a peripheric position have developed later (younger vessels) than the vessels that lie in a proximal (central) position. As a consequence, these arterioles have not disconnected from the capillary plexus, yet. In addition, several cis-trans veins (V) are developing or have already developed. In the latter case, a slight bright area is observable, too (b), which is consistent with the lesser extent and later appearance of venous (compared to arterial) disconnection.

mately remain constant, due to the conservation of the number of expelled endothelial cells (no apoptosis), their incorporation into the neighboring capillaries must create a twofold increase of these capillaries' surface. Such an increase results in a twofold increase of their radius, ${ }^{4}$ which,

\footnotetext{
${ }^{4}$ The surface of a tube of length $l$ and radius $r$ is $S=2 \pi r l$. At constant length, a twofold increase in the tube surface thus implies a twofold increase of its radius.
} 

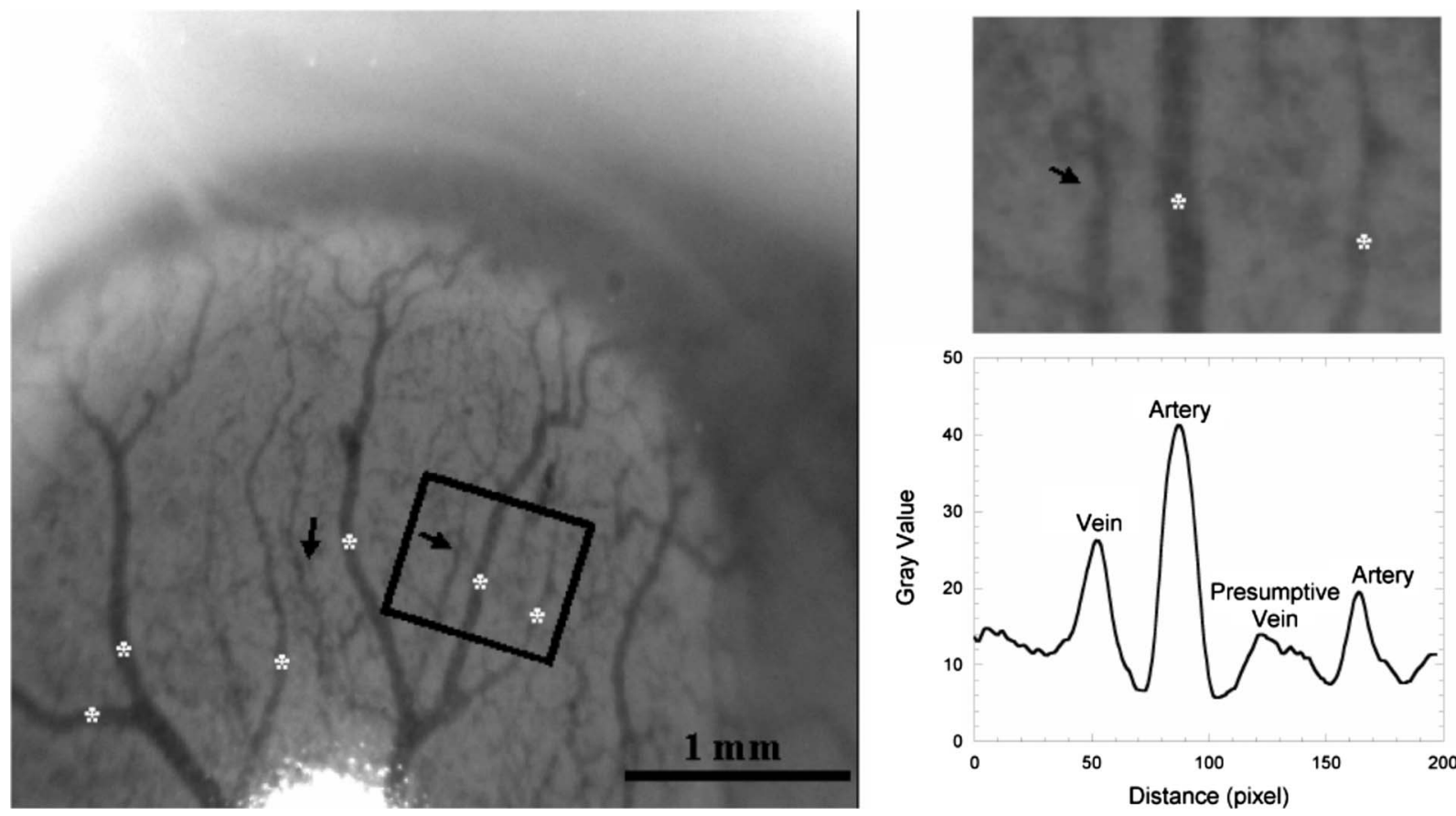

(a)
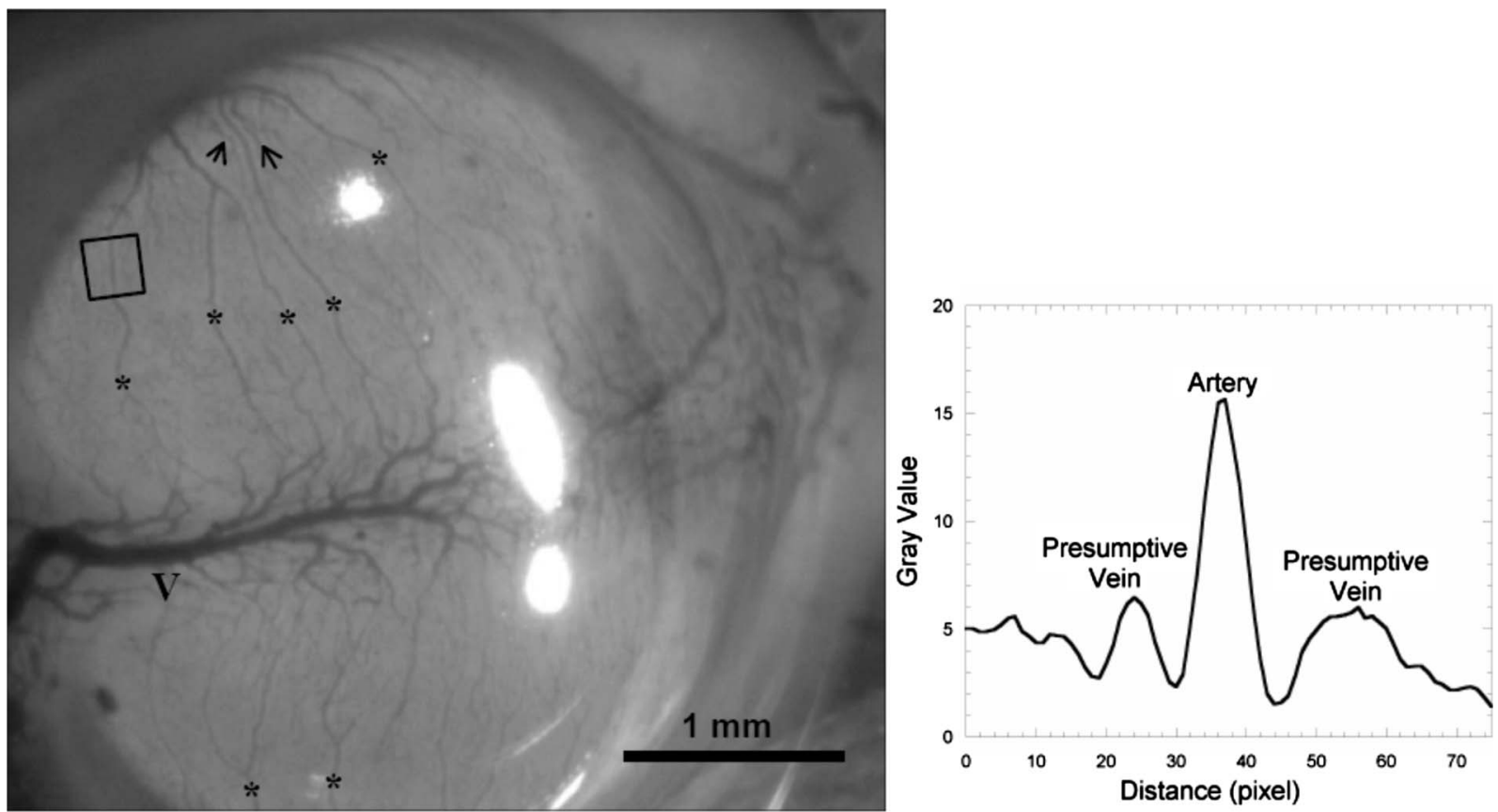

(b)

FIG. 13. Brain vessels configuration during the development of the cis-trans venous pattern. (a) Left: direct in vivo detection of the cis-trans configuration formation. The favored paths created by the remodeling of the capillary tissue in the vicinity of the growing arteries appear in the midbrain of a chicken embryo by day 5. Along the existing arteries (stars), an avascular region has appeared (see below). An area of denser capillaries can be observed somewhat afar from the existing arteries, on both sides. Thus, venous paths form parallel to the arteries (arrows). Right, top: enlarged view of the rectangular area highlighted in the left panel. Bottom: gray-level profile of this enlarged view, averaged along the vertical direction (direction parallel to the main vessel): very close to the arteries, on both sides, a strong decrease in the gray level is observable (gray value $\sim 6$ ), demonstrating the presence of an avascular area. Further away from the central artery, bumps of gray are consistent with wider capillary lumens. The bumps maxima correspond to favored venous paths, the left one being almost formed and the right one not being recognizable as a vessel, yet. Farther away, on the left, the capillary plexus reverts to an intermediate gray level (gray value $\sim 10-12$ ), representative of vascularized regions of the nonremodeled capillary plexus. (b) Similar analysis performed on a 6-day brain, from a "polar" view. Left: the growing arteries are indicated by stars. The cis-cis venous pattern (V) is at the center of this view. Arrows are pointing to the developing cis-trans venous pattern. Right: gray-level profile, averaged along the direction parallel to the vessel of the enlarged area highlighted in the left panel: two presumptive venous paths, not being recognizable as vessels yet, are evidenced. 
according to Poiseuille's law, decreases their hydrodynamic resistance by a factor of 16 .

Therefore, the incorporation of the dangling dead ends into the neighboring capillaries creates a favored path for the blood to flow, which will favor the appearance of larger vessels there. Stated otherwise, by conservations laws in the poroelastic medium, the stress exerted by the flowing blood on the arterial wall creates a porosity gradient and induces a flux of cells whose morphogenetic consequence is to generate a vein nearby. Note that such a physical effect is completely independent of any diffusible molecule which might enhance the ability of the vessels in the vicinity of the artery to enlarge. However, why should these vessels be favored only after some time and how to evaluate this time?

\section{Influence of tissue growth}

In this section, we are going to show that the hydrodynamic resistance of the cis-trans path becomes lower than that of the cis-cis path as the organ under consideration expands. For that purpose, we first need to account for the effect of tissue growth on the capillary plexus morphology. The experimental results associated with this effect will be described in Sec. IV C 1. Subsequently, in Sec. IV C 2, simple theoretical or numerical modeling approaches will be used to analyze its consequences on the hydrodynamic resistance.

\section{Influence of tissue growth on the capillary plexus morphology}

It has been shown recently that the growth of a thin piece of tissue, such as, for example, the yolk-sac, can be modeled by a very slow viscous flow, because the elasticity equation for incompressible thin solids is formally identical to the equation of a viscous fluid flow, the longitudinal displacement (in the elasticity equation) being assimilated to the fluid velocity (in the viscous fluid-flow equation) $[19,22]$. In other words, the growing elastic tissue in the yolk-sac behaves like a fluid because the yolk-sac is a thin layer: its expansion amounts to the Poiseuille's flow of a thin layer, such that the centrifugal growth of the yolk-sac, from proximal to distal areas, is associated with a pressure drop (the pressure being defined as in Sec. IV B). Since this flow is away from the yolk-sac center to its periphery, the yolk-sac center is at higher pressure (more stressed) than its edge. ${ }^{5}$ In general terms, a proximal to distal growth is associated with a gradient of pressure, such that the expansion rate is positive in the direction of the decreasing pressure.

As a consequence, in the same way as in the immediate neighborhood of the growing arteries (see Sec. IV B), but at larger scale (the scale of the yolk-sac), this pressure gradient induces a gradient of the pore-space volume: capillaries are squeezed like sponges around the yolk-sac center because of the compression exerted by the growing tissue, while they are less squeezed at its edge. This can be experimentally

\footnotetext{
${ }^{5} \mathrm{By}$ analogy, think of the trunk of a tree: if all the cells try to grow at the same rate, the center of the tree is more stressed than the edge.
}

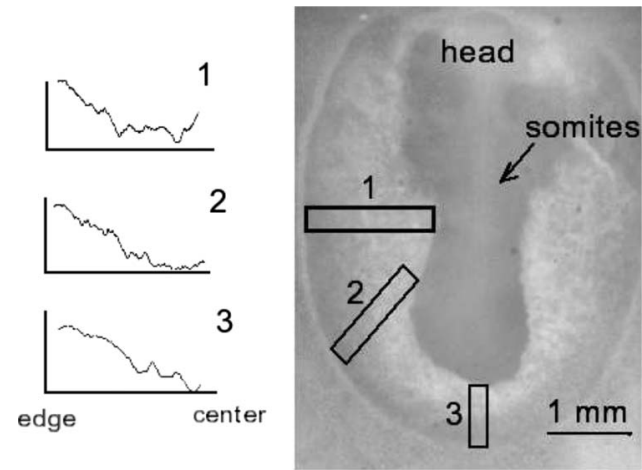

FIG. 14. Direct inspection of the capillary lattice of a 2-day embryo showing the variation in vascular density from the proximal to distal direction. Averaging over a few lines shows a regular increase of the gray level corresponding to a gradient of vascular density from the yolk-sac center to its edge. This is consistent with narrow capillary lumens at the yolk-sac center (high pressure) and larger lumens at its edge (low pressure).

confirmed by direct inspection of the capillary lattice (see Fig. 14), which demonstrates a positive gradient of capillary density from the yolk-sac center to its periphery.

\section{Influence of a gradient of capillary density on the network hydrodynamic resistance}

Due to this gradient of capillary density observable in a growing tissue, the hydrodynamic resistivity by unit length of the capillary plexus is small distally and large proximally. As a consequence, if one considers an idealized piece of growing tissue with prescribed arterial entry and venous exit points located at some distance along the proximal part, the pattern of resistances is as schematized in Fig. 15. At least for the early stages of growth, this landscape favors a flow that loops in the growing tissue and tends to explore the distal part, which is less resistive. In this situation, the total resistance of the flow loop can be roughly estimated theoretically. Let us call $R$ the large proximal resistivity by unit length between the entry and the exit and $r$ the small distal resistivity by unit length; let us call $L(t)$ the length of the growing tissue at time $t$ and $l_{0}$ the distance between the arterial and venous ends. The resistance between the vascular entry and exit points along the cis-cis path can be evaluated by

$$
r l_{0}+2[(R+r) / 2] L(t) .
$$

In this expression, the first term represents the distal capillary path and the second term represents the sum of the back and forth paths between the proximal and distal ends of the tissue (see Fig. 15), where the average resistivity by unit length is $(r+R) / 2$. Not surprisingly, as the tissue grows, the resistance to reach the distal region increases. At this very early stage of growth, where no artery is still present in the capillary plexus, the cis-trans resistance would simply be $R l_{0}$, the resistance of the shortest path from the entry to the exit points. This resistance is larger than the resistance of the cis-cis loop as long as 

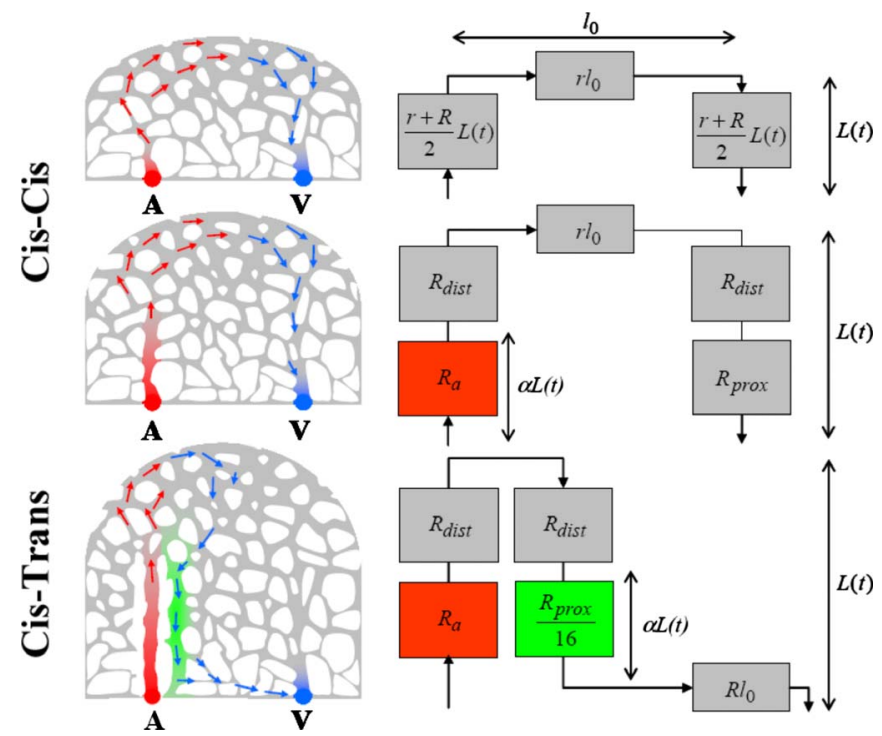

FIG. 15. (Color online) Left: schematic representation of the flow in a plexus which grows from the proximal to distal direction, before the apparition of large veins, for prescribed arterial entry (A) end venous exit $(\mathrm{V})$ points. The vessels' lumens are represented in gray and the interstitial tissue in white. The enlarged capillaries in the vicinity of the growing artery are represented in green. Arrows indicate the preferential flow path, which tends initially to explore the distal region because the capillary density is greater there. Red arrows correspond to arterial flow, blue arrows to venous flow. Right: electrical analogy for the distribution of the resistances of the preferential flow path. The upper-left "Cis-cis" panel shows the initial situation where no artery is formed yet. The cis-cis configuration provides the lowest hemodynamic resistance and thus the preferential path for blood flow. The middle panel shows the situation at start of arterial growth, with the cis-cis configuration still providing the preferential path for flow. The lower-left panel "Cistrans" shows the situation after some time of growth with the cistrans configuration providing the preferential path for flow. The (small) resistivity per unit length in the distal area and the (large) resistivity per unit length in the proximal area are denoted by $r$ and $R$, respectively. The total resistance of the proximal path (path parallel to the artery), the total resistance of the distal path (path from the arterial end to the organ distal limit), and the resistance of the growing artery are denoted by $R_{\text {prox }}, R_{\text {dist }}$, and $R_{a}$, respectively. As the artery grows, it disconnects from the capillaries. The neighboring capillaries reshape and enlarge, so that a favored path of capillaries, with their resistance divided by a factor of order 16, appears along the growing artery $\left(R_{\text {prox }} / 16\right)$.

$$
L(t)<l_{0}(R-r) /(R+r)
$$

Thus, at early stages of tissue growth, the flow loop favors capillary remodeling in the proximal to distal direction.

As the remodeling process is initially favored from the proximal to distal direction, the artery naturally grows in this direction. In addition, as this artery grows, it disconnects from the capillaries. The neighboring capillaries reshape and enlarge as described in Sec. IV B, so that a favored path of capillaries, with their resistivity divided by a factor of order 16 , appears along the growing artery. The resistances $R_{C C}$ of the cis-cis and $R_{C T}$ of the cis-trans configurations can be roughly evaluated as a function of the size $L$ of the growing organ by

$$
R_{C C}(L)=R_{a}+r l_{0}+2 R_{\text {dist }}(\alpha)(1-\alpha) L+R_{\text {prox }}(\alpha) \alpha L
$$

and by

$$
R_{C T}(L)=R_{a}+R l_{0}+2 R_{\mathrm{dist}}(\alpha)(1-\alpha) L+\frac{R_{\mathrm{prox}}(\alpha)}{16} \alpha L .
$$

In these expressions, $R_{a}$ is the resistance of the growing artery, $R_{\text {prox }}$ is the total resistivity by unit length of the proximal path (path parallel to the artery), $R_{\text {dist }}$ is the total resistivity by unit length of the distal path (path from the artery end to the organ distal limit), and $\alpha L(t)$ represents the length of the growing artery, $\alpha$ being a factor of order 1, but smaller than 1 accounting for the delay between arterial formation and tissue growth; see Fig. 15. As a consequence, the cis-cis configuration is initially much less resistive than the cis-trans configuration $(r<R)$, but it becomes resistive at a larger rate than the cis-trans configuration because $R_{\text {prox }}(\alpha)$ is greater than $R_{\text {prox }}(\alpha) / 16$. Thus, from Eqs. (3) and (4), there exists a size $L_{\max }$, given by

$$
L_{\max }=\frac{16}{15} \frac{(R-r)}{\alpha R_{\text {prox }}(\alpha)} l_{0},
$$

for which the resistances $R_{C C}\left(L_{\max }\right)$ and $R_{C T}\left(L_{\max }\right)$ of the two paths are equal.

Finally, in order to estimate $L_{\max }$, the resistivity $R_{\text {prox }}$ must be evaluated by averaging the plexus resistivity along the proximal path-i.e., for the height $h$ varying from 0 to $\alpha L(t)$. Assuming a linear decrease of the resistivity $\rho$, such as $\rho(h)=(r-R) / L(t) h+R$, we have

$$
R_{\text {prox }}(L)=\frac{r-R}{2} \alpha+R
$$

and, thus,

$$
L_{\max } \sim 2 \frac{(R-r)}{(r-R) \alpha^{2}+2 R \alpha} l_{0} .
$$

For larger sizes, the cis-trans configuration is favored.

Note that $L_{\max }$ increases with $R$ and $l_{0}$ and decreases with $\alpha$. Thus, this simplified analysis shows three important facts: the cis-cis to cis-trans transition occurs later if the entry and exit points are farther away, if the delay between arterial formation and tissue growth is longer, and if the proximal capillaries are more squeezed due to a faster growth rate. A fast growth will induce a very high proximal resistance $(R$ $\gg r$ ) and the transition will appear for $L \sim 2 l_{0}$, but a slow growth, for which, for example, $r \sim R / 3$, will induce a transition for $L \sim l_{0}$ and the cis-trans path will be preferred sooner. In this latter case, the transition length is of the order of the distance between the entry and exit points. That is to say that slow growing buds or appendages will undergo this transition as soon as they reach a roughly square or round shape: if they elongate more, the normal vascular bed must be the cis-trans one.

Note that this qualitative analysis, which is sufficient to explain the transition between the cis-cis and cis-trans configurations before the appearance of large veins, is justi- 

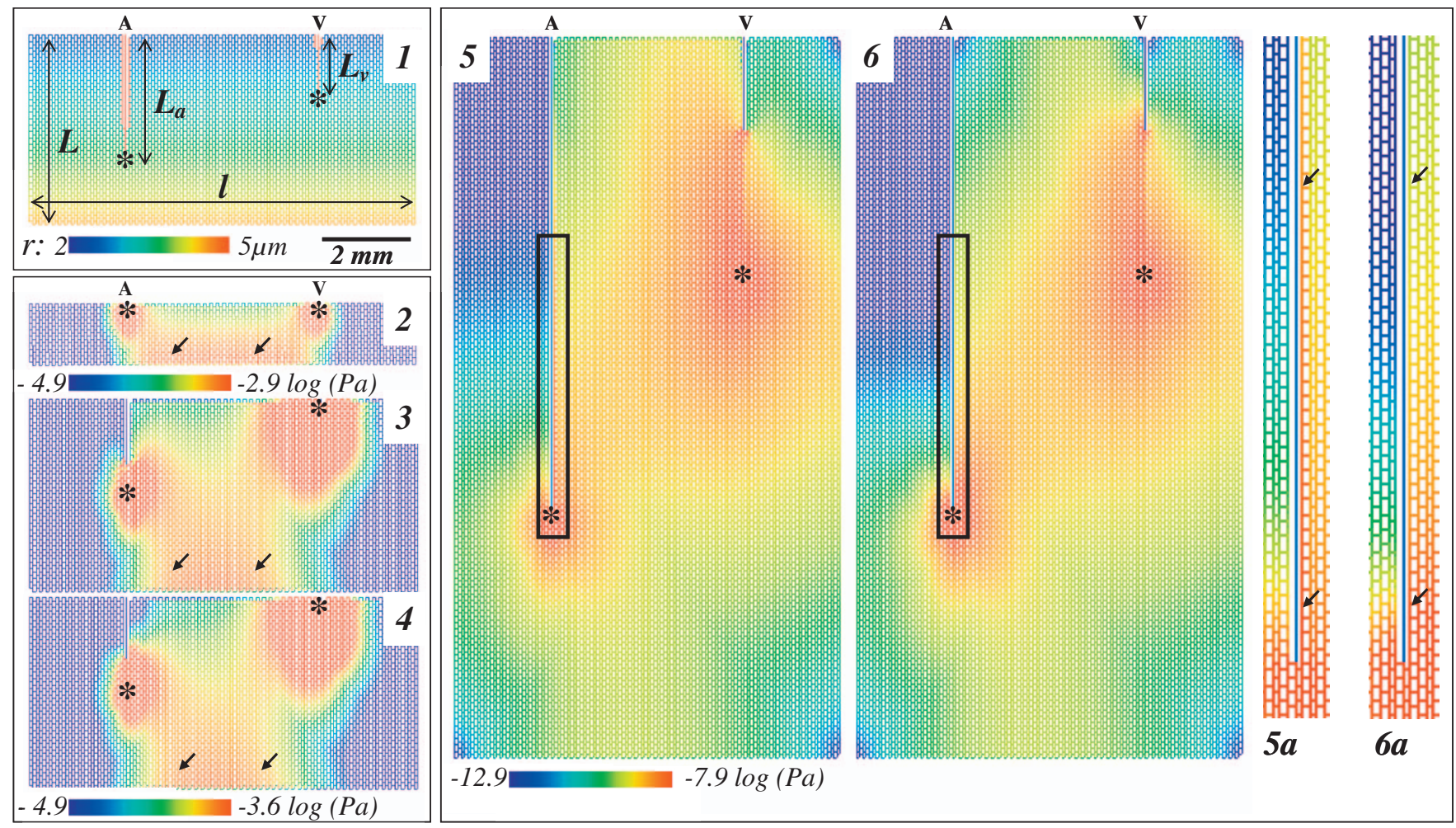

FIG. 16. (Color) Numerical simulations of the wall shear-stress spatial distribution in a model configuration representing a bidimensional growing organ at different steps, for prescribed arterial entry (A) and venous exit (V) points. Panel 1: geometry and notation: an artery (length $L_{a}$ and radius $40 \mu \mathrm{m}$ ) and a vein (length $L_{v}$, radius $40 \mu \mathrm{m}$ ) in a rectangular heterogeneous capillary plexus (length $L$, width $l$ $=7.3 \mathrm{~mm}$ ). The radius distribution of the vessels is represented by the color scale and varies linearly from $2.5 \mu \mathrm{m}$ proximally to $5 \mu \mathrm{m}$ distally. The vessels with a larger radius (including the artery and vein) appear in red. The stars indicate the end points of the artery and vein. Note that, in this case, the radius of the capillaries along the disconnected artery and vein has been increased twofold in order to account for capillary remodeling. Panels 2-6: distribution of wall shear stress: The distribution of wall shear stress (in Pa) is represented by the logarithmic color scale (note that the scale is different for the different values of $L$ in the different panels). Panel $2: L=1.2 \mathrm{~mm}$ and $L_{a}$ $=L_{v}=0.24 \mathrm{~mm}$. Panels 3 (accounting for capillary remodeling along the disconnected arteries and veins) and 4 (reference case without capillary remodeling): $L=3.6 \mathrm{~mm}, L_{a}=1.8 \mathrm{~mm}$, and $L_{v}=0.24 \mathrm{~mm}$. Panels 5 (accounting for capillary remodeling along the disconnected arteries and veins) and 6 (reference case without capillary remodeling): $L=13.5 \mathrm{~mm}, L_{a}=9 \mathrm{~mm}$, and $L_{v}=4.5 \mathrm{~mm}$; Panels $5 \mathrm{a}$ and $6 \mathrm{a}$ : enlarged views of highlighted areas of panels 5 and 6. Panels 2-4: for $L<l$, the wall shear stress exhibits a cis-cis configuration, with a high level of shear in the distal area (between arrows), favoring a proximal to distal growth of the artery and vein. At this stage, the capillary remodeling along the disconnected arteries and veins has a negligible influence (no significant differences between panel 3 and 4 ). Panels 5 and 6: For $L>l$, the wall shear stress exhibits a cis-trans configuration, with a higher level of shear in the capillaries neighboring the artery compared to the shear level in the distal area (bottom of the panel). At this stage, the capillary remodeling has an important local effect, increasing the order of magnitude of the wall shear stress in the remodeled capillaries (along the artery) by one to two orders of magnitude (area between arrows in the enlarged view displayed on panels 5a and 6a).

fied by the observed delay between the formation of arteries and veins. However, it fails to explain how and why the cis-cis loop should be unstable once large cis-cis veins have developed. That is why we give now a rationale, based on numerical simulations of the wall shear-stress spatial distribution in an idealized rectangular vascular network with prescribed arterial entry and venous exit points located at some distance along the proximal part, for what happens in this case.

The vessels growth process is governed, among other things, by the spatial distribution of pressure in the capillary plexus, which is inherently nonlocal; i.e., the pressure at a given point in space is influenced by the pressure distribution in the entire capillary plexus, and not only by the pressure distribution in its immediate neighborhood: in the simplest case of an homogeneous and isotropic capillary plexus, the pressure spatial dependence satisfies the Laplace's equation. Thus, all the details of the vascular geometry, including the capillary plexus, the arterial, and venous trees, must be considered in order to analyze the flow field, particularly the wall shear-stress distribution, in the growing organ. In addition, small local variations in the geometry such as local remodeling may have a significant effect on this distribution. To date, a complete mathematical treatment of this problem cannot be performed in complex situations where an already developed tree exists and is still growing.

Therefore, in order to study the flow patterns as a function of the length $L$ of the growing tissue once large cis-cis veins have developed, numerical simulations of the wall shearstress spatial distribution in an idealized bidimensional rect- 
angular vascular network exhibiting a positive gradient of capillary density from its center to its periphery have been performed. Such a gradient of capillary density was imposed by prescribing a gradient in capillary radius (see Fig. 16, panel 1 , for the imposed geometry). The influence of the remodeling of the capillary plexus in the vicinity of the disconnected arteries and veins has also been studied by prescribing, or not, a twofold increase of the radius of their neighboring capillaries.

Corroborating the simple theoretical results presented above, we have found that the cis-cis path is initially favored, without any significant influence of the capillary remodeling along the disconnected arteries and veins. Indeed, if the length of the growing tissue is smaller than its width $(L<l)$, the wall shear stress exhibits a cis-cis configuration, with a high level of shear in the distal area (between arrows in Fig. 16, panels 2-4), favoring a proximal to distal growth of the artery and vein, and a flow looping across the distal capillary plexus. At this stage, the capillary remodeling along the disconnected artery and vein has a negligible influence (no significant differences between panels 3 and 4). On the contrary, if the length of the growing tissue is larger than its width $(l<L)$, the capillary remodeling along the disconnected artery has an important local effect, increasing the wall shear stress in the remodeled capillaries by one to two orders of magnitude (Fig. 16, area between arrows in panels $5 \mathrm{a}$ and $6 \mathrm{a}$, representing enlarged views of highlighted areas of panels 5 and 6). This result is not trivial because, in a tube of radius $r$, the wall shear stress scales as $Q / r^{3}, Q$ being the flow rate: for a twofold increase in radius, the flow rate must increase by two to three orders of magnitudes to allow the observed increase of the wall shear stress. It demonstrates that capillary remodeling along the disconnected arteries has a positive feedback for the selection of the cis-trans configuration. In other words, due to the capillary remodeling along the disconnected artery, a favorable flow route is selected along this growing artery, high shear stresses leading to a further enlargement of the vessels of this flow route. In the absence of such a capillary remodeling, one might still expect the growth of a second venous tree, because the wall shear stress exhibits a cis-trans configuration, with a higher level of shear in the capillaries located between the arterial and venous extremities (stars in panel 5) compared to the shear level in the distal area (bottom of the panels). However, this second venous tree would develop only showing a weak (statistical) pairing with the artery, as numerically observed in $[17,19]$. Conversely, the moderate increase of lumen diameters close to the artery suffices to induce the growth of a parallel vein, allowing to state that the "artery induces its vein."

Of course, we only present a static case (no self-consistent growth, no self-consistent evolution of arterial entry and venous exit points), whereas the vessels growth process is a highly dynamic one, the vessels being permanently remodeled to adapt to the local flow conditions. However, the selection of a favorable flow route along a disconnected artery at a given time will lead to an accelerating enlargement of this flow route, with a further amplification of the effects. First, the formation of a second set of veins adjacent to the artery will lead to the modification of venous exits, with the appearance of additional exit points for the cis-trans flow. Such a modification will further reduce the hydrodynamic resistance of the cis-trans path (by eliminating the unphysical venous flow path represented at the bottom of Fig. 15, left panel, where arterial entry and venous exit points were prescribed). This will further facilitate the selection of the cistrans path. Second, the formation of this second set of veins will lead to a flow decrease in the initial set of veins, which may lead, or not, to their regression, depending on the size of the considered organ and on the growth speed. For example, in the forelimbs, the two set of veins coexist: the superficial veins, which can be observed through the skin, are not paired with an artery; the deep veins accompany the arteries and constitute the venae comitantes of those vessels. In these fast growing organs, which are more elongated than the yolk-sac or the brain, the chronological formation of the brachial artery (i.e., the main artery of the forelimb between shoulder and elbow) and vein as well as the evolution of the anterior and posterior marginal veins and marginal sinus (which lay at the forelimb periphery and which may be considered as the equivalent of the sinus vein in the yolk-sac) is indeed in full accordance with our analysis. This chronology can be deduced from a recent study [23] which depicts in great details the vascular development in the forelimb of the quail embryo.

According to this study, the cis-cis to cis-trans transition takes place between stages $\mathrm{HH} 26$ and $\mathrm{HH} 29 .^{6}$ The size of the forelimb at $\mathrm{HH} 26$ can be roughly evaluated from Figs. 3B and $3 \mathrm{~F}$ from [23] (ventral views) to be $2.5 \mathrm{~mm}$ in length and $1-1.15 \mathrm{~mm}$ in depth-i.e., $L \sim 2.2 l_{0}-2.5 l_{0}$-which is very close to our prediction for a fast-growing organ.

\section{DISCUSSION}

As indicated in the Introduction, a largely open problematic in the field of vascular development is understanding the relative contribution of intrinsic (molecular) programming

\footnotetext{
${ }^{6}$ The relevant chorological elements are summarized as follows: Stage HH21. The arterial branch of the dorsal artery passing to the forelimb in the space between the 18th and 19th somites has enlarged to become the largest artery supplying the forelimb. It is referred to as the primary subclavian artery ( $\left.1{ }^{\circ} \mathrm{SCA}\right)$; Stage HH23. Peripheral vessels along the postaxial (posterior) border of the forelimb in capillary layer III have begun to form the posterior marginal vein (PMV). Stage HH25. The centrally placed $1^{\circ} \mathrm{SCA}$ is the largest blood vessel in the forelimb and is now referred to as the brachial artery (BA). The anterior marginal vein (AMV) has begun to form. Stage HH26. The posterior marginal vein joins the anterior marginal vein to form the marginal sinus (MS). Stage HH29. The width of the MS decreases in the apex of the forelimb, but the AMV and the PMV still constitute the major pathways of venous drainage. At the junction of the zeugopod and the stylopod in the cubital fossa (elbow), the AMV and the PMV pass from the subcutaneous plane into the developing deep brachial vein. This vein develops parallel to the brachial artery. Stage HH32. The MS continues to degenerate, and the connections of both the AMV and the PMV with the brachial vein become prominent. The AMV and the PMV are now denoted as the cephalic vein and basilic veins, respectively.
} 
and extrinsic (physiological or epigenetic) factors on the morphological evolution of the vascular system. The main result of our work is that, during vertebrate development, arteries exert a morphological control over the venous pattern through extrinsic factors. Thus, a fine biochemical regulation of arterioveinous cross talk is not required for the arteries to influence the venous patterning.

This result could seem in contradiction with published evidence of the importance of molecular programming in the early arteriovenous differentiation of endothelial cells. Indeed, a tremendous amount of work, based on the discovery of distinct molecular markers for arterial and venous endothelial cells, has been conduced in a last few years to elucidate the genetic mechanisms driving such a differentiation. In this context, Zhong et al. [24] have demonstrated an early fate restriction of individual endothelial precursors to either an arterial or a venous identity and not both: the whole lineage of endothelial cells originating from one of these precursors is subsequently incorporated in the same type of vessels. Other evidence for a direct arterial and venous specification occurring prior to blood flow have been reviewed in $[25,26]$. In particular, a biochemical growth factor, called the vascular endothelial growth factor (VEGF), is believed to play a critical role in triggering the other genetic pathways known to be involved in this specification, such as the Notch pathway. These findings have been interpreted in favor of a genetic determination of endothelial cell identity, which could seem in contradiction with the present work. However, the detailed mechanisms underlying the early induction of the endothelial identity remain unclear [26,27] and the signaling pathways that drive arterial differentiation and that lie upstream of VEGF are still unknown.

In addition, other studies have demonstrated that endothelial cell identity determined by genetic factors is reversible and is finally modified by hemodynamics. Transplanted endothelial cells of arterial or venous origin can integrate into both arteries and veins in host embryos, and independent of their original identity, these incorporated cells express arterial or venous markers identical to the host vessels $[28,29]$. Furthermore, manipulation of blood flow in chick embryos affects endothelial cell identity after the circulation initiates: ligation of a vitelline artery in the chick embryo by a metal clip morphologically transforms an artery into a vein, marked by downregulation of arterial markers and upregulation of venous markers. Restoration of arterial flow by removing the clip restores arterial marker expression [4]. These results highlight the ability of mechanical factors to induce a phenotypic change of endothelial cells toward an arteriolar or a venous fate.

As far as we know, mechanical factors have not been ruled out as one of the signaling mechanisms lying upstream of the VEGF that could drive arterial differentiation before the onset of flow. It has even been demonstrated that mechanical stimuli such as fluid shear stress or external stretch can stimulate VEGF protein production, both in vitro [30] and in vivo [31]. Because we have shown that tissue stress is a key determinant of vascular patterning ([19] and present work), it would be interesting to seek for a potential effect of tissue stress on the early arterial and venous specification. Indeed, tissue expansion, even in the absence of any flow, already induces mechanical forces. Therefore, as mechanical forces may induce a phenotypic change of endothelial cells toward an arteriolar or a venous fate, this may as well happen before or after the onset of flow. After the onset of flow, it is important to keep in mind that the cis-cis to cis-trans transition induces an extremely interdigitated stress field, such that endothelial cells located very close to each other may feel very different pressure values or shear stresses. From a material science point of view, a vascular bed is a composite, poroelastic medium.

Nevertheless, if adopting such a point of view can lead to a better understanding of the mechanisms of embryonic development, it is clear to us that proper molecular programming remains a necessity in vascular morphogenesis. Indeed, the molecular specificities of the endothelial cells are determinant for defining the interactions between them and with their environment, as well as their reactions and plasticity in response to mechanical stresses (mechanotransduction).

Finally, an indirect consequence of the present work may be of practical importance: the interpretation of yolk-sac remodeling abnormalities in mice deficient for genes implicated in vascular development must be extremely careful and account for the modifications in perfusion (for example due to cardiac malformations) as well as in growth rate due to the targeted deletion of the gene. Indeed, part of the vascular phenotype observed in the yolk-sac of these null mice can in fact be due to these modifications, which are quite frequent [32-37]. In the same spirit, the origin of several developmental abnormalities, such as the Holt-Oram syndrome [38] and even the complete absence of limbs, might have to be reconsidered. Indeed, these abnormalities have been related to the misexpression or mutation in the developmental gene Tbx5 ([38] and references therein). However, this gene is associated with early developmental abnormalities of the heart and sometimes to hypoplasia of the vessels of the upper limbs. Therefore, the absence of limb development in these syndromes might either be a direct consequence of vascular abnormalities or a consequence of abnormal mechanotransduction, leading to abnormalities in both the heart and limbs.

\section{CONCLUSION}

The physiological formation of a vascular system exhibits a striking spatiotemporal feature: veins first develop in series with the arteries, while after some time, which depends on the organ considered, new veins arise which are parallel to the existing arteries (arteries and vein form two juxtaposed, yet distinct, trees), although they are still downstream in terms of flow. In this paper, we have shown how such a transition is physically possible, and why it may be expected with generality, as organisms grow. First, the capillary plexus of a growing piece of tissue is not uniform. The distal regions have larger vascular (capillary) densities and, hence, a smaller hydrodynamic resistance. As a consequence, the first flow route passes through distal regions (cis-cis configuration). As the arteries grow into this piece of tissue, they get disconnected from the capillary plexus, which induces an excluded region around them. Second, the capillary path neighboring the disconnected arteries is remodeled and its 
hydrodynamic resistance is significantly reduced. Therefore, another favored flow path emerges along these arteries. As a consequence, a second flow route emerges (cis-trans configuration) when the piece of tissue extends. Between the cis-cis and cis-trans configurations, all configurations are in principle possible, and self-organization of the vessels will contribute to determining their exact pattern. However the global aspect-cis-cis, cis-trans, or in between-will depend on the size at which the growth is arrested and on the growth rate (or, in other words, on the stress gradient in the growing tissue). This complex spatiotemporal feature, which is rarely emphasized in the literature, is probably at the origin of a great confusion in anatomic descriptions which sometimes depict paired vessels and sometimes unpaired vessels or which ascribe the cis-cis to cis-trans transition to the opening of a particular vessel in the embryo-for example, the opening of the duct of Cuvier. Actually, since this transition is observed in several quite different organs, a general mechanism has to exist, independently of ad hoc developmental explanations. We believe that the mechanism described here is more general and applies to other organs than the yolk-sac and the brain, which were taken here as case studies.

\section{ACKNOWLEDGMENTS}

This work has been partly supported by ACI Technologies Pour la Santé, Grant No. 02 TS 031, of the French Department of Education, Research and Technology, by a grant from La Fondation de France, and by a grant from the French cancer research association, ARC.
[1] S. Isogai, N. D. Lawson, S. Torrealday, M. Horiguchi, and B. M. Weinstein, Development 130, 5281 (2003).

[2] D. Ribatti, J. Anat. 208, 139 (2006).

[3] V. Fleury, M. Unbekandt, A. Al-Kilani, and T.-H. Nguyen, Organogenesis 3, 49 (2007).

[4] F. Le Noble, D. Moyon, L. Pardanaud, L. Yuan, V. Djonov, R. Matthijsen, C. Bréant, V. Fleury, and A. Eichmann, Development 131, 361 (2004).

[5] S. C. Chapman, J. Collignon, G. C. Schoenwolf, and A. Lumsden, Dev. Dyn. 220, 284 (2001).

[6] F. A. Le Noble, N. H. Schreurs, H. W. van Straaten, D. W. Slaaf, J. F. Smits, H. Rogg, and H. A. Struijker-Boudier, Am. J. Physiol. 264, 460 (1993).

[7] H. M. Evans, Anat. Rec. 3, 498 (1909).

[8] W. Risau, Nature (London) 386, 671 (1997).

[9] V. Djonov, O. Baum, and P. H. Burri, Cell Tissue Res. 314, 107 (2003).

[10] A. Eichmann, L. Yuan, D. Moyon, F. Le Noble, L. Pardanaud, and C. Bréant, Int. J. Dev. Biol. 49, 259 (2005).

[11] R. Thoma, Untersuchunung über die Histogenese und Histomechanik des Gefässystems (Ferdinand Hencke Verlag, Stütgart, 1893).

[12] T. C. Skalak and R. J. Price, Microcirculation 3, 143 (1996).

[13] A. R. Pries, T. W. Secomb, and P. Gaehtgens, Am. J. Physiol. 275, H349 (1998).

[14] V. Fleury and L. Schwartz, Fractals 7, 33 (1999).

[15] A. L. Romanoff, The Avian Embryo (Macmillan, New York, 1967).

[16] W. J. Hacking, E. Van Bavel, and J. A. Spaan, Am. J. Physiol. 270, H364 (2003).

[17] V. Fleury and L. Schwartz, Fractals 8, 255 (2000).

[18] H. U. Wang, Z. Chen, and D. J. Anderson, Cell 93, 741 (1998).

[19] T.-H. Nguyen, A. Eichmann, F. Le Noble, and V. Fleury, Phys. Rev. E 73, 061907 (2006).

[20] T.-H. Nguyen (unpublished).

[21] Ph. A. Charlez, Theoretical Fundamentals, Vol. 1 of Rock Mechanics (Editions Technip, Paris, 1991).

[22] V. Fleury, Organogenesis 2, 6 (2005).

[23] D. Bates, G. I. Taylor, and D. F. Newgreen, Dev. Biol. 249,
300 (2002)

[24] T. P. Zhong, S. Childs, J. P. Leu, and M. C. Fishman, Nature (London) 414, 216 (2001).

[25] N. D. Lawson and B. M. Weinstein, Nat. Rev. Genet. 3, 674 (2002).

[26] J. Torres-Vázquez, M. Kamei, and B. M. Weinstein, Cell Tissue Res. 314, 43 (2003).

[27] M. Hirashima and T. Suda, Endothelium 13, 137 (2006).

[28] D. Moyon, L. Pardanaud, L. Yuan, C. Breant, and A. Eichmann, Mech. Dev. 106, 133 (2001).

[29] K. Othman-Hassan, K. Patel, M. Papoutsi, M. RodriguezNiedenfuhr, B. Christ, and J. Wilting, Dev. Biol. 237, 398 (2001).

[30] Y. T. Shiu, J. A. Weiss, J. B. Hoying, M. N. Iwamoto, I. Suk Joung, and C. T. Quam, Crit. Rev. Biomed. Eng. 33, 431 (2005).

[31] I. Rivilis, M. Milkiewicz, P. Boyd, J. Goldstein, M. D. Brown, S. Egginton, F. M. Hansen, O. Hudlicka, and T. L. Haas, Am. J. Physiol. 283, H1430 (2002).

[32] P. Carmeliet, M. G. Lampugnani, L. Moons, F. Breviario, V. Compernolle, F. Bono, G. Balconi, R. Spagnuolo, B. Oostuyse, M. Dewerchin et al., Cell 98, 147 (1999).

[33] N. V. Iyer, L. E. Kotch, F. Agani, S. W. Leung, E. Laughner, R. H. Wenger, M. Gassman, J. D. Gearhart, A. Lawler, A. L. Yu et al., Genes Dev. 12, 149 (1998).

[34] O. Krüger, A. Plum, J. S. Kim, E. Winterhager, S. Maxeiner, G. Hallas, S. Kirchhoff, O. Traub, W. H. Lamers, and K. Willecke, Development 127, 4179 (2000).

[35] H. E. Ryan, J. Lo, and R. S. Johnson, EMBO J. 17, 3005 (1998).

[36] L. D. Urness, L. K. Sorensen, and D. Y. Li, Nat. Genet. 26, 328 (2000).

[37] S. S. Gerety and D. J. Anderson, Development 129, 1397 (2002).

[38] T. Bossert, T. Walther, J. Gummert, R. Hubald, M. Kostelka, and F. W. Mohr, http://www.orpha.net/data/patho/GB/ukHOS.pdf

[39] T. L. Labus and J. C. Aydelott, NASA Technical Note NASA-TN D6368 (unpublished). 